\title{
Mechanistic Modeling of Ophthalmic Drug Delivery to the Anterior Chamber by Eye Drops and Contact Lenses
}

Samuel Gause, Kuan-Hui Hsu, Chancellor Shafor, Philip

\author{
Dixon, Kristin Conrad Powell, Anuj Chauhan*
}

Department of Chemical Engineering; University of

Florida; Gainesville, FL 32611

*Corresponding author: Email: chauhan@che.ufl.edu, Tel: (352) 392 2592; fax: (352) 3929513 


\section{Abstract}

Ophthalmic drug for the anterior chamber diseases are delivered into tears by either eye drops or by extended release devices placed in the eyes. The instilled drug exits the eye through various routes including tear drainage into the nose through the canaliculi and transport across various ocular membranes. Understanding the mechanisms relevant to each route can be useful in predicting the dependency of ocular bioavailability on various formulation parameters, such as drug concentration, salinity, viscosity, etc. Mathematical modeling has been developed for each of the routes and validated by comparison with experiments. The individual models can be combined into a system model to predict the fraction of the instilled drug that reaches the target. This review summarizes the individual models for the transport of drugs across the cornea and conjunctiva and the canaliculi tear drainage. It also summarizes the combined tear dynamics model that can predict the ocular bioavailability of drugs instilled as eye drops. The predictions from the individual models and the combined model are in good agreement with experimental data. Both experiments and models predict that the corneal bioavailability for drugs delivered through eye drops is less than $5 \%$ due to the small area of the cornea in comparison to the conjunctiva, and the rapid clearance of the instilled solution by tear drainage. A contact lens is a natural choice for delivering drugs to the cornea due to the placement of the contact in the immediate vicinity of the cornea. The drug released by the contact towards the cornea surface is trapped in the post lens tear film for extended duration of at least 30 min allowing transport of a large portion into the cornea. The model predictions backed by in vivo animal and clinical data show that the bioavailability increases to about $50 \%$ with contact lenses. This realization has encouraged considerable research towards delivering ocular drugs by contact lenses. Commercial contacts are, however, not ideal for drug delivery due to the short release durations 
which may necessitate wearing multiple lenses each day, reducing the viability of this approach. Recent research has focused on designing contacts that retain all critical properties while increasing the release durations to a few hours or a few days. Beagle dog studies with contact lenses containing vitamin E nanobarriers to attenuate drug transport have shown promising results. Human studies using contacts for drug delivery have also been conducted for allergy therapy but drug eluting contacts are not available in the market for any therapy.

Keywords: Contact lenses; Eye drops; Drug delivery; Mechanistic models; Cornea

\section{Outline}

1. Introduction

2. Modeling drug transport across the multi-layer cornea

2.1 Strucutre

2.2 Compartment models

2.3 Mechanistic models

2.4 Penetration kinetics of lipophilic drugs

2.5 Penetration kinetics of hydrophilic drugs

3 Modeling drug transport across the conjunctiva

3.1 Structure

3.2 Model

3.3 Model validation

4 Modeling drainage of tears from eyes to nose

4.1 Mechanism

4.2 Model 


\subsection{Model validation}

5 Tear Dynamics Model

5.1 Model

5.2 Model validation

6 Ocular pharmacokinetics for delivery based on eye drops

6.1 Model

6.2 Model validation

6.3 Deficiencies of drug delivery by eye drops

7 Drug delivery by contact lenses

7.1 Modeling dispersive mixing in the POLTF

7.2 Model Validation

7.3 Modeling drug delivery by contacts

7.4 Model Validation

7.5 Limitation of commercial contacts for drug delivery

7.6 Approaches for improving drug release

8 Conclusions 


\section{Introduction}

Delivery of ophthalmic drugs to treat diseases of the anterior chamber is challenging in spite of the excellent accessibility and the potential for targeting by delivering drugs directly into the tears. Drugs delivered topically into the tears face multitude of transport barriers and clearance pathways that can lead to very low bioavailability, i.e., the fraction of the instilled drug that reaches the target tissue [1]. Specifically, eye drops account for about $90 \%$ of ophthalmic medications for front of the eye diseases but the corneal bioavailability for drugs delivered via eye drops is less than 5\% [2]. This review focuses on summarizing the relevant transport mechanisms for ophthalmic drug delivery while focusing on mathematical models for each of the transport process and then integrating those into a tear dynamics model that can predict the pharmacokinetics of drugs delivered into the eyes. While several prior reviews have summarized recent advances in ophthalmic drug delivery, including designing novel formulations and devices to overcome the deficiencies of eye drops [3-7], this paper presents quantitative models that can be valuable in understanding the dynamics of anterior-segment drug delivery and designing optimal delivery devices. Posterior segment drug delivery, which is becoming increasingly important due to the growing number of patients is not considered in this paper. The models described here are based on mass balances for the fluid and drugs introduced into the tears either as a bolus through eye drops or by an extended release from a drug-eluting device placed in the eye. The mass balances include fluxes of the fluid and the drugs through various pathways of the ocular epithelia, including the corneal epithelia as well as the bulbar and palpebral conjunctiva (Figure 1). Since we are focusing here on drug delivery for treatment of the anterior segment diseases, the key quantity of interest is the mass of drug transported across the cornea. 
This review focuses on integrating the physiology and anatomy of the human eye into mathematical models for drug transport. A detailed knowledge of ocular anatomy and physiology are not required to follow the models, but a brief description (presented below) is useful. A human tear film, illustrated in Figure 1, contains about 6-7 $\mu \mathrm{l}$ of fluid [8] divided into three compartments: the precorneal tear film, the conjunctival sac, and the tear menisci which contains a majority of the tears. The tears are bounded by the corneal epithelium and the conjunctiva epithelia, including both the bulbar and the palpebral, which is about seventeen times the area of the cornea [9]. Drug introduced into the eyes exits the tear volume through transport across the cornea, palpebral conjunctiva, or the bulbar conjunctiva-sclera, and additionally through drainage of the tears into the nose through the canaliculi. Thus, the drugs instilled in the tears reach the targeted intraocular tissues by taking either the corneal pathway or bulbar conjunctival-scleral pathway (Figure 1). The latter route is crucial to some specific drugs $[10,11]$, but is generally regarded as non-productive because the conjunctiva is highly vascularized and a high fraction of the drug diffusing through this membrane is flushed away into systemic circulation by transport into blood capillaries [2,12]. Most ophthalmic drugs take the corneal pathway, which penetrates through the multi-layer cornea structure [1]. The mechanisms of drug transport across the cornea and the conjunctiva are thus central to understanding the ocular pharmacokinetics. Additionally, drugs exit the tear volume as tear fluid which is continuously drained from the eyes to the nose during blinking, contributing to reduced bioavailability.

This paper is organized to first discuss relevant mechanisms, and then describe mathematical models. Sections 2 and 3 present models for the transport of drugs across the multi-layer cornea and the conjunctiva, respectively. Section 4 describes the model for the tear 
drainage from the eye. Next, we present a model for tear dynamics in Section 5 and apply that to estimate the corneal bioavailability for drugs delivered through eye drops in Section 6. The deficiencies of eye drops become evident through the model predictions, motivating the following section which focuses on drug delivery via contact lenses (Section 7).

\section{Modeling drug transport across the multi-layer cornea}

2.1 Structure - A basic knowledge of the anatomy of cornea is required to understand the mechanisms of drug transport. Principally, the cornea is stratified into three layers which are, from the outside to the inside of the eye, the epithelium, the stroma, and the endothelium. These layers are separated by Bowman's and Descemet’s membranes (Figure 2). In a human cornea, the epithelium is mainly composed of cells with tight junctions and can be further stratified into another 5-7 layers with a total thickness of about 40-50 $\mu \mathrm{m}$. This layer exhibits significant resistance to the transport of hydrophilic drugs due to its high lipid content and the multistranded tight junctions in between the cells. The resistance to lipophilic drugs is relatively small because the drugs can readily dissolve in the lipid bilayers of the plasma membrane and transport through diffusion without significant partitioning into the cells. The second layer, the stroma, is a hydrophilic layer that contains about $80 \%$ water with a thickness of $450-500 \mu \mathrm{m}$ that accounts for $90 \%$ of the total thickness of the cornea [13]. This water layer in turn creates significant resistance to lipophilic drugs due to solubility and partition coefficient limitation, with minimal resistance for transport of hydrophilic drugs. Finally, the endothelium is a leaky cellular monolayer with a thickness of roughly $13 \mu \mathrm{m}$ and provides little resistance to paracellular transport of drugs [14].

2.2 Compartment models are frequently used to model drug transport in the cornea even though such models neglect the heterogeneities of the multi-layer structure and assumes the 
entire cornea is a well-stirred, homogeneous compartment $[15,16]$. This oversimplified model, however, fails to provide information about the resistance encountered by the drug in each layer and whether the drug transport is taking the transcellular or paracellullar pathway. In addition, this lumped model is only valid when the concentration reaches pseudo-steady state which is not an appropriate assumption for eye drop delivery due to the short residence time.

2.3 Mechanistic models - The pharmacokinetic models for drug transport in the eye can be considerably improved by integrating the detailed structure of the cornea and accounting for all drug transport mechanisms and pathways. Including the detailed spatial heterogeneity of the corneal epithelium in models is difficult due to the limitation on spatial resolution of detection instrumentation [17]. However, macroscopic tissue based models that include average transport at the cellular scale and consider the diffusion transport in each single layer, the interfacial resistance, and the binding kinetics to the cell have been developed [17,18]. Clearly, lipophilic and hydrophilic drugs face different resistances and favor different routes (transcellular or paracellular pathway) when crossing through the cornea. The classification of drugs as hydrophilic and hydrophobic is somewhat arbitrary and there could be several drugs that may not fall in either of these two extreme categories. For such drugs a more generalized model is needed. The drugs could be classified into these two categories based on the aqueous solubilities or partition coefficients $(\log P)$ between organic solvent and water (most commonly an noctanol/water partition coefficient) [19]. In the following pharmacokinetics model, we take rhodamine ( $\log P \sim 2-2.5$ in octanol/water) [17] as examples of a lipophilic drug. We also propose a model for and fluorescein ( $\log P \sim 0$ in octanol/water [20]), which is considered as a hydrophilic compound due to high aqueous solubility but it has a finite affinity for the lipohilic pathways and thus both paracellular and transcellular mechanisms may need to be included. 
2.4 Penetration kinetics of lipophilic drugs - Lipophilic drugs instilled in the tears transport across the cornea by first partitioning and diffusing through the lipid bilayers of the epithelium cells. Next, the transport across the hydrophilic stroma is likely diffusive, followed by transport through the endothelium, which is similar in structure to the epithelium. Measurements of the dynamic concentration profiles of rhodamine, a hydrophobic fluorescent molecule, show that the mechanisms described above are broadly accurate (Figure 3). Rhodamine is infused from the tear film (from left-hand side in Figure 3) at a constant concentration and accumulates in the epithelium to a relatively high concentration in a time scale of a few hours. The concentration in the stroma drops promptly due to the solubility limitation and forms a small peak in the endothelium. The fluorescence profiles show distinct discontinuities in concentration at the boundaries between epithelium, stroma and endothelium, which suggests the adequacy of dividing the cornea into three layers in the model. Noteworthy is that the slow accumulation of rhodamine in the epithelia can be attributed to the transport from the lipid bilayers to the "intracellular lipophilic domains" such as the endoplasmic reticulum, which is rate limited by transportation through the cell membrane and the hydrophilic cytoplasm (Figure 4). The transient mass balance of the drugs is thus described as (see equation 1 in [17])

$\frac{\partial C_{L, 1}}{\partial t}=D_{L, 1} \frac{\partial^{2} C_{L, 1}}{\partial y^{2}}-k_{L, 1}\left(C_{L, 1}-\frac{C_{L, 1}^{b}}{K_{L, 1}}\right)$ Eq. 1a

$\frac{\partial C_{L, 1}^{b}}{\partial t}=k_{L, 1}\left(C_{L, 1}-\frac{C_{L, 1}^{b}}{K_{L, 1}}\right)$ Eq. $1 b$ where $t$ is time, $C_{L, 1}$ represents the concentration of drug in epithelial bilayers (where the subscript $L$ denotes lipophilic drugs and 1 refers to the epithelium layer), $C_{L, 1}^{b}$ represents the concentration in the intracellular lipophilic domains, and $K_{L, 1}$ is the ratio of $C_{L, 1}^{b}$ and $C_{L, 1}$ at 
equilibrium. $D_{L, 1}$ is the effective diffusivity of the lipophilic drug in epithelial bilayers, $k_{L, 1}$ is the permeability for the pseudo-steady transport across the cytoplasm from the lipid bilayers to the cell interior, and $y$ is the depth across the cornea with $y=0$ at the interface of the tear film and the epithelium. This model can be considered as a multi-scale model as it integrates the cellscale transport into the overall transport through the epithelium. The mechanics of the celltransport are, however, grossly simplified in this model. For example, influx (facilitate cell permeation) and efflux (restrict cell permeation) transporters are identified in the corneal epithelium, which contribute to overall corneal permeation to various extents [21-23]. Further improvements could be achieved by using a more detailed analysis and considering both the passive (driven by concentration gradient) and the active (carrier-mediated) transport of drug across the bilayers to the interior of the cells. The effective diffusivity through the bilayers considered in this model is an average diffusivity that accounts for the true diffusivity, tortuosity, as well as the barrier to transport from one cell to the next. Due to the similarity between the epithelium and endothelium, the transport through the endothelium can be modeled in the same manner as the epithelium (Eq. 1a and 1b), but using the subscript L,3 to indicate lipophilic drugs in the endothelium:

$\frac{\partial C_{L, 3}}{\partial t}=D_{L, 3} \frac{\partial^{2} C_{L, 3}}{\partial y^{2}}-k_{L, 3}\left(C_{L, 3}-\frac{C_{L, 3}^{b}}{K_{L, 3}}\right)$ Eq. 2a

$\frac{\partial C_{L, 3}^{b}}{\partial t}=k_{L, 3}\left(C_{L, 3}-\frac{C_{L, 3}^{b}}{K_{L, 3}}\right)$

The transport in stroma was described by the diffusion equation with an effective diffusivity that is a function of the binding of the solute to the components of the stroma:

$\frac{\partial C_{L, 2}}{\partial t}=D_{L, 2} \frac{\partial^{2} C_{L, 2}}{\partial y^{2}}$ Eq. 3 
Essentially the binding of the drugs to collagen fibrils and glycosaminoglycans (GAGs) in the stroma is a relatively rapid event compared to diffusion time scales and so the free and the bound concentration are in equilibrium [24].

Appropriate boundary conditions including continuity of flux and local equilibrium at each boundary can be assumed to solve the above equations. First, it is assumed that the concentration of drug readily reaches equilibrium at the interface of tear film and epithelium, so that

$C_{L, 1}(y=0)=\varphi_{L, 10} C_{L, 0}(y=0)$ Eq. 4

where the subscript 0 indicates the tear film and $\varphi_{L, 10}$ is the partition coefficient of the drug between epithelium and tear. The concentration equilibrium condition at the interface of epithelium and stroma is described as

$D_{L, 1} \frac{\partial C_{L, 1}}{\partial y}=k_{L, p e r m}\left(C_{L, 1}-\frac{C_{L, 2}}{\varphi_{L, 21}}\right)$ Eq. 5

where $k_{L, \text { perm }}$ is the permeability of epithelium-stroma interface and $\varphi_{L, 21}$ is the partition coefficient of the drug in stroma and epithelium. The permeability was introduced into the model because the experimental data showed that the ratio of the epithelium and stroma concentrations at the boundary changed with time. Physiologically, the origin of the barrier was not clear. In addition, from flux continuity, we know

$D_{L, 1} \frac{\partial C_{L, 1}}{\partial y}=D_{L, 2} \frac{\partial C_{L, 2}}{\partial y}$ Eq. 6

Similarly, the boundary conditions at the stroma-endothelium interface are

$D_{L, 2} \frac{\partial C_{L, 2}}{\partial y}=D_{L, 3} \frac{\partial C_{L, 3}}{\partial y}$ Eq. 7 
Finally, the concentration of drug at the interface of endothelium and aqueous humor would also reach equilibrium so that

$C_{L, 4}=\varphi_{L, 43} C_{L, 3}$ Eq. 9

where the subscript 4 indicates the aqueous humor and $\varphi_{L, 43}$ is the partition coefficient of the drug in endothelium and aqueous humor. This model was verified using the experimental data for transient concentration profiles of rhodamine B across an excised rabbit's cornea, measured using a custom-built confocal scanning fluorescence microscope [17]. The fittings (solid lines in Figure 3) were robust and the values of the fitted parameters were in good agreement with estimations based on scalings and prior measurements. As suggested by the model and experimental data, the overall rate limiting step for the slow accumulation is transport through the hydrophilic cytoplasm within the cells. This accumulation is however irrelevant on shorter time scales typically associated with drug delivery by eye drops due to the brief residence time of the eye drops in the eyes.

2.5 Penetration kinetics of hydrophilic drugs - Gupta et al. [18] measured the dynamic concentration profiles of a hydrophilic molecule, fluorescein (MW: 376), after infusing an excised rabbit cornea from the anterior chamber side, using a custom-built confocal scanning fluorescence microscope to measure the concentration across the cornea. Representative concentration profile changes with time are shown in Figure 5. Fluorescein is diffusing from the anterior chamber, through the endothelium, stroma and epithelium to reach the tear chamber, as evident from the decreasing concentrations towards the tears. At long times, the concentration profile in the stroma increases towards the epithelium which was ascribed to the combined 
effects of convection and diffusion. The pressure on the anterior chamber side was kept higher to maintain the shape of the cornea and that resulted in a convective flow through the cornea. The positions of endothelium-stroma and epithelium-stroma interfaces are indicated by the arrows in "Zoom A" and "Zoom B", respectively (Figure 5). Similar to the transport of hydrophobic molecules, the fluorescence profiles show distinct discontinuities in concentration at the layer interfaces, and thus the cornea is again viewed as a three-layer structure in the model. Fluorescein accumulation in stroma is expected based on its hydrophilic nature, but the molecule also accumulates in endothelium and epithelium. Since fluorescein is hydrophilic and small in size, it is expected to primarily diffuse through the epithelium and endothelium by taking the paracellular path because of the high resistance offered by the lipid bilayers for the transcellular path (Figure 4). Certain hydrophilic drugs could transport through the cell layers as a result of active transport but this mechanism appears to be not relevant to fluorescein as evident from the very slow accumulation into the epithelium. The gradual accumulation in epithelium and endothelium suggests the transcellular route could be important at long periods. Thus, the transport of fluorescein in the endothelium is modeled as

$\begin{array}{ll}V_{3} \frac{\partial C_{H, 3}}{\partial t}=p_{H, 3} A\left(C_{H, 4}-\frac{C_{H, 3}}{\varphi_{H, 34}}\right)-p_{H, 3} A\left(\frac{C_{H, 3}}{\varphi_{H, 32}}-C_{H, 2}\right) & \text { Eq. } 10\end{array}$

where the subscript $H, 2,3$ and 4 indicates hydrophilic molecules, stroma, endothelium and anterior chamber, respectively; $\varphi_{H, 34}$ is the partition coefficient of the drug between the cytoplasm of the endothelium and the anterior chamber and $\varphi_{H, 32}$ is the partition coefficient between the cytoplasm of the endothelium and the stroma; $p_{H, 3}$ is the permeability of the endothelial lipophilic membranes; $V_{3}$ and $A$ are the volume of endothelium and the cross area available for transcellular transport. As in the model for lipophilic drugs, the detailed molecule 
transport through the cell membrane is grossly simplified and is considered as a whole in the permeability term. The boundary condition at the interface of endothelium and stroma can be described by flux continuity or further simplified to

$C_{H, 3}=\varphi_{H, 32} C_{H, 2}$ Eq. 11

which implies that the concentration readily reaches equilibrium at the endothelium-stroma interface.

For a diffusion-controlled system, we know that the diffusion time scales as diffusion distance squared. From Figure 5, we can see the fluorescein takes 42 minutes to reach a depth of $175 \mu \mathrm{m}$ into the stroma but less than 81 minutes to reach the full thickness of the stroma ( 350 $\mu \mathrm{m})$. The rapid transportation rate in the stroma is clearly not diffusion-controlled, at least in this in vitro experimental setup. Therefore, the transport of fluorescein in the stroma is described by a convection-diffusion model

$\frac{\partial C_{H, 2}}{\partial t}+v \frac{\partial C_{H, 2}}{\partial y}=D_{H, 2} \frac{\partial^{2} C_{H, 2}}{\partial y^{2}}$ Eq. 12

where $v$ is the convective velocity in stroma. The inclusion of convection term can also explain the change in the direction of concentration gradient at long times. As in the model developed for lipophilic drugs, the binding to collagen and GAGs in the stroma was assumed to occur rapidly and the effective diffusivity takes into account the binding.

Due to the significant resistance encountered in the epithelium, the concentration flux from stroma to epithelium is essentially negligible in short time ( $<200$ minutes in Figure 5$)$. Thus the boundary condition at the interface of stroma and epithelium is described as

$$
-\mathrm{D} \frac{\partial C_{H, 2}}{\partial y}+v C_{H, 2}=0
$$


The evolution of concentration in the epithelium takes place over a long time scale of a few hrs to days. Thus it is consistent to use a zero flux condition at the epithelium interface to solve for the concentration profiles in the stroma and then use the predicted long term solutions to model the concentration transients in the epithelium. Based on the experimental data, the accumulation occurs on a time scale of a few hours to a day, again suggesting that this is not relevant when drugs are delivered via eye drops due to the short residence times of a few min.

This model was validated by fitting to the experimental data which used fluorescein as the hydrophilic solutes transported across a rabbit's cornea [18]. The fittings (solid lines in Figure $5 B$ ) were robust and the values of the fitted parameters were in good agreement with estimations based on scalings or prior measurements. Note that the positions of epithelium-stroma and stroma-endothelium interfaces are opposite to that in Figure 5A. Based on the model and experimental observation, the concentration in stroma reaches equilibrium faster than that in endothelium and epithelium, which suggests that fluorescein can transport relatively readily through paracellular pathway across the tight junction. However, as the contact time of solute to the endothelium and the epithelium increases, the transcellular pathway becomes more important because the solutes are accumulating slowly in the cell of the endothelium and epithelium. It is noted that a major limitation of this model is the neglect of active transport, which must be included for drugs that are known to utilize transporters in diffusing across the lipid bilayers. The slow accumulation of fluorescein in the epithelium was fitted to lump transport model to yield a permeability of $0.92 \times 10^{-8} \mathrm{~m} / \mathrm{s}$. This suggests that the 40 micron thick epithelium will equilibrate with the stroma concentration in about a day. Since the eye drops remain on the surface for only a few min, transport of any hydrophilic drugs into the epithelium can be safely neglected. Indirect evidence for this phenomena was recently provided by Hsu et al. [25] from 
animal studies focusing on continuous delivery of glaucoma drugs using contact lenses. This study utilized a vitamin E modified contact lens that provided continuous release of a combination of hydrophilic glaucoma drugs, timolol and dorzolamide, for up to 96 hours. The animal studies on glaucomatous Beagle dogs showed that the therapeutic effects did not disappear, but sustained for more than a week after the drug-eluting contact lenses were removed (Figure 6). This was attributed to the creation of drug depots in epithelium due to the slow transport of the drug into the cells while the contacts are in place. After the contacts are removed, the drug transported into the cells, diffuses out towards the target tissue providing the extended pharmacodynamics effect.

\section{Modeling drug transport across the conjunctiva}

3.1 Structure -The bulbar and palpebral conjunctiva are made up of several layers of epithelial and goblet cells which separate the tear film from blood vessels. Studies examining guinea pigs by Nichols determined that the epithelial cells are responsible for the transport of ions and water [26] while the secretion of the mucus layer is a more complex process. Goblet cells secrete gel forming mucins which give the tear film many of its rheological properties while the lacrimal gland produces smaller mucins which help lubricate and protect the eye $[27,28]$. In addition to these, most apical surface epithelial cells express mucin on their surface creating a protective glycocalyx layer [29]. Shedding of these mucin from the surface also contributes to the dissolved mucin in the tear film. Transport across the epithelial cells is accomplished by taking paracellular pathways or ion channels, water channels, cotransporters, and transport pumps for ion, glucose, and water located in the cell membrane (a simplified version can be seen in Figure 7) [30,31]. Solutes diffusing into the palpebral conjunctiva will eventually reach the blood stream while solutes diffusing into the bulbar conjunctiva can either transport into the 
capillaries to reach the systemic circulation or diffuse into the sclera and then into the intraocular tissue. Due to the highly vascularized nature of the bulbar conjunctiva, the vast majority of solutes end up in the blood stream [1].

3.2 Model - Conjunctiva plays an important role in maintaining the physiological concentrations of ions in the tear film. Additionally, the conjunctiva could secrete tears into the eyes through a combination of osmotic and electro-osmotic transport. The tear secretion into the eyes and the drug transport from the tears into the conjunctiva play important roles in the pharmacokinetics of drug instilled as eye drops. Assuming that solutes diffusing into both the bulbar and palpebral conjunctiva eventually reach the blood, both of these can be lumped together. The transport mechanisms in the conjunctiva are complex due to the specific transporters and ion channels. Like cornea, conjunctiva is multilayer, but the transport is rate limited by the first layer of cells contacting the tear film [32]. Zhu et al. developed model for transport of ions, solutes and water across the conjunctiva accounting for both transcellular and paracellular diffusion and also various ion channels and transporters [33]. Incorporating these specific transporters is important because the transport of ions leads to osmotic and potential gradients across the conjunctiva, which in turn impact the water secretion. Specifically, for the conjunctival epithelium, the transport of ions is dominated by $\mathrm{Na}^{+}$-glucose cotransporters and $\mathrm{Cl}^{-}$ channels on the tear film side cell membrane and by $\mathrm{Na}^{+}-\mathrm{K}^{+}$pumps, $\mathrm{Na}^{+}-\mathrm{K}^{+}-\mathrm{Cl}^{-}$cotransporters, and $\mathrm{K}^{+}$channels on the blood vessel side [30,31]. The rate equations for fluxes through the ion channels and transporters are available in literature and were directly applied to model the transport in conjunctiva without any additional parameters. The water transport is assumed to be due to osmotic flow through the transcellular and paracellular pathways, along with electroosmotic flow through the paracellular pathway. The various transport mechanisms (passive 
transcellular and paracellulr diffusion, ion channels, specific transporters, electrophoretic and electroosmotic fluxes) were included into the mass balances to compute the concentrations of various components inside the cells and the net transport across an excised conjunctiva subjected to a gradient of composition or potential. In addition, the lacrimal sac secretes tear fluid containing what is assumed to be a constant concentration of each solute while drainage through the canaliculi must be taken into account and is modeled using the methods shown in Section 4 of this paper. It is assumed that the concentration of each solute in the blood vessels is not affected by transport from the tear film and is thus a known constant. An illustration of the model assumptions is given in Figure 7.

Using these assumptions, it is possible to determine mass balances for solutes in the tear film and in the cellular compartment. The mass balances for the ions in the tear film and in the cell can be generalized by the following equations (see Eqs. 1-4 and 6-9 in [33])

$$
\begin{aligned}
& \frac{d\left(C_{i, \text { tear }} V_{\text {total }}\right)}{d t}=q_{\text {secretion }} c_{i, \text { tear }}^{0}-q_{\text {drainage }} c_{i, \text { tear }}-\left(J_{i, \text { channel }}+J_{i, p a r a c e l l u l a r}\right) S_{\text {conj }} \\
& \frac{d\left(C_{i, \text { cell }} V_{\text {cell }}\right)}{d t}=\left(\sum J_{i, \text { channel }} S_{\text {conj }}\right)-r_{i} c_{i, \text { cell }}
\end{aligned}
$$

and the water balance for the system is given by (see Eqs. 5 and 10 in [33])

$$
\begin{array}{ll}
\frac{d V_{\text {tear }}}{d t}=q_{\text {secretion }}-q_{\text {drainage }}-q_{\text {evaporation- }}\left(J_{w, \text { paracellular }}+J_{w, \text { transcellular,tear }}\right) S_{\text {conj }} & \text { Eq. 3a } \\
\frac{d V_{\text {cell }}}{d t}=\left(J_{w, \text { transcellular , tear }}-J_{w, \text { transcellular , blood }}\right) S_{\text {conj }} & \text { Eq. 3b }
\end{array}
$$

where $\mathrm{c}_{\mathrm{i}, \text { tear }}$ is the concentration of solute $\mathrm{i}$ in the tears, $\mathrm{V}_{\text {total }}$ is the total tear volume, $\mathrm{q}_{\text {secretion }}$ is the generation of tear fluid by the lacrimal and conjunctiva sacs, qevaporation is the evaporation rate of the water in the tear film, $\mathrm{c}^{0} \mathrm{i}$,tear is the concentration of solute $\mathrm{i}$ in the secreted tear fluid 
(assumed to be a constant), $\mathrm{q}_{\mathrm{drainage}}$ is the flow rate of tears out of the eyes through the canaliculi (discussed at length in Section 4), $\mathrm{J}_{\mathrm{i} \text {,channel }}$ is the flux of solute i through a channel in the cell membrane (if such a channel exists for the solute), $\mathrm{J}_{\mathrm{i} \text {,paracellular }}$ is the flux of solute $\mathrm{i}$ through the paracellular path, $\mathrm{J}_{\mathrm{w} \text {,transcellular,tear }}$ is the flux of water across the tear side cell membrane, $\mathrm{J}_{\mathrm{w} \text {,transcellular,blood }}$ is the flux of water across the blood vessel side cell membrane, $\mathrm{J}_{\mathrm{w} \text {,paracellular }}$ is the flux of water through the paracellular pathway, $\mathrm{S}_{\text {conj }}$ is the area of the conjunctiva, $\mathrm{C}_{\mathrm{i} \text {,cell }}$ is the concentration of solute $\mathrm{i}$ in the cell, $\mathrm{V}_{\text {cell }}$ is the volume of the cells, and $\mathrm{r}_{\mathrm{i}}$ is the rate of consumption of solute i in the cell (typically only applies to glucose). Eqs. 2 and 3 can be solved along with the condition of electroneutrality (i.e. the net flux of ions in and out of the tear film and the cell is zero) to find the concentration of each solute over time and the total volume of water in each section.

3.3 Model validation - Typical experiments to determine transport properties across the conjunctival epithelia involve mounting excised epithelium between Ussing chambers and determining the short-circuit current $\left(\mathrm{I}_{\mathrm{SC}}\right)$, the potential difference across the epithelia (PD), or the fluxes of various components added to the donor compartment [30,31]. The model for the transport across the conjunctiva was validated by comparing the fluxes of various ions after mounting an excised conjunctiva membrane in a diffusion cell. Model predictions for chloride and sodium ion fluxes were both within $30 \%$ of the experimental measurements [33]. The validity of the model was also tested by predicting the dynamic changes in short-circuit current after making certain modification to the properties of the conjunctiva. For instance, one can block the ion channels, pumps, or co-transporters by use of certain chemicals, which will impact the net flow of charges across the conjunctiva. The new steady state $\mathrm{I}_{\mathrm{SC}}$ and the time to reach $50 \%$ of this change $\left(t_{1 / 2}\right)$ are useful in determining the effectiveness of the model in transient and 
steady state conditions. For the transient conditions when specific pumps, channels, or cotransporters were inhibited, most $t_{1 / 2}$ values were lower for the model than for the experimental data. For example, when the Na-K pump was inhibited, the experimental data showed a $t_{1 / 2}$ of 41.5 min while the model predicted 18.57 min. This decrease in expected transient times can be explained by the fact that in the Ussing chamber experiments, chemicals need to be added to the chamber in order to block these channels. In experiments, these chemicals could take time to become well mixed in the system and start working, while the model assumes that they are instantly well mixed and effective [33]. For steady state conditions, the model predicted the change in $\mathrm{I}_{\mathrm{SC}}$ to within $30 \%$ of the experimental results for most of the cases, supporting the validity of the model.

\section{Modeling drainage of tears from eyes to nose}

4.1 Mechanisms - Studies have shown that the drainage of the tear film from the eye is an active process that is dependent on blinking [34,35]. Tears drain from the eyes to the nose through canaliculi, which are a pair of channels that connect the tear film to the lacrimal sac (Figure 8c) [36]. The canaliculi open into the tear film through circular openings called puncta (Figure 8c). A blink cycle can be divided into the blink, during which the eyes close and then open, followed by the interblink, which is the period of time in between two successive blinks. At the beginning of a blink, the canaliculi are filled with tears. Then, as the lid closes, the puncta are occluded closing the contact of the canaliculi to the tear film. At the same time, the muscles surrounding the canaliculi apply a compressive force on the canaliculi which creates a pressure gradient to push the tear fluid into the lacrimal sac. Thus, the canaliculi compress to a smaller radius and tears flow towards the nose during the blink. Once the lid begins to open, the pressure in the canaliculi drops as the muscles surrounding it relax. When the eyelid is approximately 
halfway open, the puncta separate and burst open, pulling tear fluid from the tear film meniscus into the canaliculi. During this phase, the lacrimal sac opening is closed, preventing drainage of tears from the eye during the interblink phase [37]. Thus, during the interblink, the size of the canaliculi increases due to the fluid drawn from the tear film. This pumping action between the blink and interblink periods drives the drainage of tears from the eye. This sequence is illustrated in Figure 8.

4.2 Model - The model for the tear drainage is based on combining the deformation of the elastic walls of the canaliculus with equations for fluid flow through the canaliculus (Figure 8). A local force balance taking into account the external pressure applied by the muscles and the hoop stress developed due to the deformation of the canaliculi wall is (see eq. A1 in [38])

$-R L\left(p_{\text {out }}-p\right)=b L\left(E_{c} \frac{R-R_{0}}{R_{0}}\right)$

Eq. 4

where $\mathrm{p}_{\text {out }}$ is the pressure applied to the outside of the walls of the canaliculi, $\mathrm{p}$ is the pressure in the fluid inside, $\mathrm{R}$ is the radius, $\mathrm{R}_{0}$ is the undeformed radius, $\mathrm{L}$ is the length (assuming the canaliculi is a single, straight tube), and $\mathrm{b}$ and $\mathrm{E}_{\mathrm{c}}$ are the thickness and the modulus of the canaliculus, respectively. This force balance can be coupled to the Poiseuille flow through the canaliculus to yield (see eq. 1 in [38])

$\frac{b E_{c} R_{0}}{16 \mu} \frac{\partial^{2} R}{\partial x^{2}}=\frac{\partial R}{\partial t}$ Eq. 5

where $\mu$ is the viscosity of the tear fluid, $\mathrm{x}$ is the distance along the canaliculi (with $\mathrm{x}=0$ at the puncta and $x=L$ is the opening to the lacrimal sac), and $t$ is the time. The boundary conditions for this model can be deduced by considering the physiology of the ocular blink cycle. In the blink, the two puncta occlude each other implying that the flow rate at $x=0$ is zero. The 
pressure at the nasal end of the canaliculi can be assumed to be atmospheric during the blink. In the interblink, the puncta open which allows equilibration of pressure in the canaliculi with that in the tear film. The pressure in the tear film is set by the meniscus curvature, which can be directly measured by imaging or related to the total tear volume through geometric constraints. Finally, the flow in the canaliculi at the nasal end is zero due to the pinning of the contact line. The differential equation can be solved along with these boundary conditions (see eqs. 2-3 in [38]) to yield the time and position dependent radius of the canaliculus and thus, the canaliculus volume. The rate of change of volume of the canaliculus represents the drainage rate of tears from the eyes to the nose. Based on this model the volume change of the tear film due to drainage, for a complete blink-interblink cycle is simply the change in volume of the canaliculi chamber during this cycle. Assuming the timescales for the blink and interblink phases are each sufficient to reach an equilibrium radius, the drainage of tears from the eye for each blink can be given by (see eq. 7 in [38])

$q_{\text {drainage }}=\frac{\Delta V}{\Delta t}=\frac{\Delta\left(\pi R^{2} L\right)}{\Delta t}=\left(\frac{\pi L}{t_{c}}\right)\left(R_{i b}^{2}-R_{b}^{2}\right)$ Eq. 6

where $t_{c}$ is the time for a complete blink-interblink cycle and $\mathrm{R}_{\mathrm{ib}}$ and $\mathrm{R}_{\mathrm{b}}$ are the equilibrium radii (calculated from Eq. 4) for the interblink and blink phases, respectively. For the interblink phase, the muscles are not pushing on the canaliculi resulting in a pressure of zero from outside the canaliculi walls. The pressure inside is equal to the pressure in the meniscus outside the puncta, which is simply a function of the surface tension and the radius of the meniscus. For the blink phase, the pressure outside is assumed to be equal to $\mathrm{p}_{0}$, a constant pressure that is applied due to the muscles pushing on the canaliculi, while the pressure inside (at steady state) is equal to the pressure in the lacrimal sac that the canaliculi drain into [38]. This gives the following equations for the equilibrium radii (see eq. 4 and 5 in [38]) 
$R_{b}=\frac{R_{0}}{1+\frac{\left(p_{0}-p_{s a c}\right)}{b E_{c}}}$

Eq. $7 \mathrm{a}$

$R_{i b}=\frac{R_{0}}{1+\frac{\sigma}{b E_{C}} R_{0}}$

Eq. $7 \mathrm{~b}$

where $\mathrm{p}_{\mathrm{sac}}$ is the pressure inside the lacrimal sac, $\mathrm{R}_{\mathrm{m}}$ is the radius of the meniscus in the eye, and $\sigma$ is the surface tension between the tear fluid and the air.

As previously stated, Eq. 6 assumes that the canaliculus reaches an equilibrium radius during both the blink and inter blink phases. There are, therefore, three important time scales relevant to the drainage process: the duration of the blink phase $(0.04 \mathrm{~s})$, the length of the interblink phase (6 s) [37], and the time scale required to achieve an equilibrium canaliculus radius. As evident from Eq. 5 and from the analysis of Zhu et al, the time for the canaliculus to reach an equilibrium radius after the application of a pressure is $\tau_{e q} \equiv \frac{16 \mu L^{2}}{\pi^{2} b E_{c} R_{0}}$ [38]. When the tear viscosity is less than $4.4 \mathrm{mPa} \cdot \mathrm{s}, \tau_{e q}<<0.4 \mathrm{~s}$ and the assumption of an equilibrium canaliculus radius is valid for both the blink and interblink phases. For tear viscosities between $4.4 \mathrm{mPa} \cdot \mathrm{s}$ and $645 \mathrm{mPa} \cdot \mathrm{s}$, this assumption is valid for the interblink phase, but not the blink phase [39]. In this case, the canaliculus radius is not uniform at the end of the blink phase and $\mathrm{V}_{\text {blink}}$, the volume change in the canaliculi during a single blink phase, needs to be calculated by determining the position dependent radius at the end of the blink. Eq. 6 can then be used to determine the change in fluid in the canaliculi with the new blink radius. Note that for viscosities between $4.4 \mathrm{mPa} \cdot \mathrm{s}$ and $645 \mathrm{mPa} \cdot \mathrm{s}$, increasing viscosity will decrease the drainage rate and that this viscosity range is characteristic of most of the high viscosity Newtonian fluids that are used for ocular instillation. When the viscosity is larger than $645 \mathrm{mPa}$, the canaliculus 
radius cannot reach steady state during either the blink phase or the interblink phase. Further, at this high viscosity, the shearing to the ocular surface is likely to be high and may cause irritation.

4.3 Model validation - All of the parameters are known for the models presented except for $\mathrm{b}$ and $\mathrm{E}_{\mathrm{c}}$. These parameters were estimated by Zhu et al by fitting the model to experimental values for the tear outflow and tear secretion rates. The value of $b \cdot E_{c}$ determined from this method was $2.57 \mathrm{~Pa} \cdot \mathrm{m}$ [40]. Zhu et al also measured these parameters in porcine eyes to determine approximate values. They found that the elastic modulus was a function of the frequency of the applied stress. For the biologically relevant frequencies of 1-25 Hz (determined from the blink duration of $0.04 \mathrm{~s}$ and the interblink duration of several seconds), the value of $b \cdot E_{c}$ was found to range from 4.51-7.51 Pa.m [41]. These results are comparable to those used for the model, especially since these studies used porcine eyes as opposed to human eyes. This model was further validated by comparing the drainage rates and pressure transients predicted by the model to experimental values found in the literature. The measured drainage rates vary across different studies with a reasonable agreement between the prediction of 0.1-0.2 $\mathrm{mm}^{3}$ [38] and measured values of $0.58 \mathrm{~mm}^{3}$ [35] per blink phase.

Experiments performed by Wilson et al determined pressure transients in the canaliculi while blinking. These results showed that for the blink phase, the pressure starts at zero and quickly jumps to 400-533 $\mathrm{Pa}$ in $0.1-0.2 \mathrm{~s}$ after blinking began. The pressure then drops to $400-$ 533 Pa below zero in 0.1-0.2 s after the eyelids begin to open, after which it returns to zero in 0.5-0.6 s [42]. This differs slightly from the model, which predicts that the pressure will start at 167Pa before a blink and will increase to approximately $233 \mathrm{~Pa}$ within less than $0.05 \mathrm{~s}$ after the blink starts. The model then predicts that the pressure will drop to -400 Pa immediately after the blink phase followed by a rise to $-167 \mathrm{~Pa}$ within $0.05 \mathrm{~s}$. Note that experiments showed a 
significantly longer time to reach the steady state pressure for the interblink phase than for the blink phase, which is not predicted by the model. This difference can be partially accounted for by considering that the lower puncta was filled with a pressure measurement device whose large diameter prevented the puncta from connecting to the tear film. Due to this, the tear fluid cannot enter the lower canaliculus directly, but instead must travel through the upper canaliculus, thereby increasing the time required for equilibration of pressures. In addition to increasing the time to reach steady state for the interblink phase, this would change the equilibrium pressure of the lower canaliculi contributing to the observed differences between the model predictions and the experiments [38].

\section{Tear Dynamics Model}

5.1 Model - The mass balance for the tears is given by (see eq. 1 in [40])

$\frac{d V_{\text {total }}}{d t}=q_{\text {secretion }}-q_{\text {drainage }}-q_{\text {evaporation }}-q_{\text {conjunctiva }}$

Eq. 8

where, $\mathrm{q}_{\text {secretion }}$ is the rate of tears produced by the lacrimal glands that is known a priori, qdrainage is the rate of tear loss due to the canaliculi pumping action that can be estimated from the drainage model, $\mathrm{q}_{\text {conjunctiva }}$ is the rate of fluid transported across the epithelia that can be calculated from the conjunctiva transport model, and $\mathrm{V}_{\text {total }}$ is the tear volume which is the sum of the volume of exposed tear fluid on the cornea, the volume of tear fluid in the tear film meniscus, and the volume of tear fluid in the conjuctival sacs. It is critical to note that the total volume can be related to the radius of curvature through geometric considerations, and the curvature radius impacts the pressure in the film, that in turn impacts the drainage rate. This indirect relationship between the drainage rates and the tear volume is crucial in determining the steady state tear volume. The rate of evaporation $\mathrm{q}_{\text {evaporation }}$ can either be estimated based on the mass transfer 
models that account for the presence of the lipid film on the surface or can be obtained from experimental data. The transport of fluid across the conjunctiva, qconjunctiva, depends on the osmolarity and electrical potential, which in turn depend on the concentration of various ions in the tears. Thus, the tear balance equation must be supplemented by species mass balances for all relevant components, particularly the sodium, chloride, and potassium ions (see equations 1-4 and 6-9 in [33]). In this model, the transport across the cornea is neglected because the area of the conjunctiva is significantly larger than the area of the cornea, therefore fluxes across the cornea do not significantly impact the balances. The model for tear dynamics along with the individual models for drainage and transport across the conjunctiva were solved to predict the steady state tear volume and composition and the dynamics of volume and solute concentrations after instillation of eye drops. It is noted that assuming the entire tear volume to be a well-mixed single compartment is a simplification that prevents analysis of spatial variations in various regions of the tear volume. Several recent publications have built upon the models described here by including multiple compartments with finite inter-mixing and also integrating blinks into the models $[43,44]$.

5.2 Model validation - Several studies have been performed examining the removal of tracer compounds that do not penetrate the cornea or conjunctiva. Measurements of the time to reach equilibrium for the volume of the tears, the concentration of the tracer, and the quantity of the tracer after instillation of an eye drop containing this tracer compound have all been reported in the literature and can be used for comparison with this model. Yokoi and Ishibashi both measured the volume of the tear film over time in the eye after instillation of a $15 \mu \mathrm{L}$ drop. Their results show that the volume of the tear film returns to steady state conditions after 300-600 s [45,46]. The model predicted this time to be $1115 \mathrm{~s}, 2-4$ times the rate shown by experiments 
[33]. The discrepancy here can be partially explained by the fact that addition of the eye drop fluid weakens the lipid layer of the tear film, which would increase the rate of evaporation. Studies performed on rabbits showed that this could cause an increase in the evaporation rate of as much as 17 times [47]. Increasing the rate of evaporation of the tear film would reduce the time to equilibrium for the volume. For this reason, the time for removal of the drug from the system is also over predicted, with experimental results taking 900-1000 s for a tracer compound in a $25 \mu \mathrm{L}$ drop to reach $1 \%$ of its original value $[48,49]$ while the model predicts this time to be 1454 s. Again, increasing the rate of evaporation would result in the model predicting a lower value. When the rate of evaporation was set at 4 times the value originally used in the model, the time for removal of the drug decreased by 9\% [40]. Finally, the concentration of the tracer compound in the tears was also measured over time. The time after eye drop instillation to reach $1 \%$ of its initial value was determined. Experimental results showed that, for a $40 \mu \mathrm{L}$ drop, this time was $4800 \mathrm{~s}$ [50] while the model predicts this time to be $2480 \mathrm{~s}$. An explanation for this discrepancy may be found by examining the drug used. The authors of this study noted that the drug used is an irritant and may cause stimulated tearing. This stimulated tearing would increase the rate of tear secretion. The experimental and model results were comparable when the tear secretion rate was increased by a factor of 3 to account for this tearing from irritation [40].

Measurements of the concentration of a radioactive tracer introduced to the eye through eye drops showed that the concentration reached a steady state of $15 \%$ of the initial value after $360 \mathrm{~s}$, which was not predicted in the model [51]. It is possible that this discrepancy is due to penetration of the compound into the ocular tissue, as the molecule has a smaller molecular weight than the cutoff reported for the cornea and conjunctiva [40]. Additional discrepancies between model and experiments were found when examining the rate of clearance of a 
radioactive tracer. Snibson et al. found that there was a biexponential decay of the tracer compound with a rapid decay in the first 60-120 s after instillation of the eye drop [48]. Additionally, it was found experimentally that 23-50\% of the tracer compound remained after 60 s, while the model predicted this time to be 750 s and 420 s for $23 \%$ and $50 \%$ of the tracer compound remaining, respectively [40,51]. The rapid clearance seen in experiments may be due to the movement of the tracer from the ocular surface (where measurements of the tracer compound were performed) to the medial canthus (area near puncta plugs, see figure 8) and lacrimal sac. As the lacrimal sac was not distinguished from the medial canthus region, whether the decrease was due to rapid drainage or merely migration of the tracer in the eye is not known.

\section{Ocular pharmacokinetics for delivery based on eye drops}

6.1 Model - Eye drops are currently the most widely used form of drug delivery to the front of the eye [2], accounting for nearly $90 \%$ of the market for eye related medications. Many experimental studies have shown, however, that the corneal bioavailability for drugs delivered via eye drops is lower than 5\%. The tear and solute dynamics models summarized above can be used for estimating the corneal bioavailability for drug delivered through eye drops. Essentially, the tear balances yield the dynamic concentration of drug, which can then be integrated into the cornea transport model to yield the mass of drug transported into the aqueous humor. It is noted that neglecting the cornea transport in the overall tear balance is supported by the experimental data for very low bioavailability of drugs delivered via eye drops. Integrating the multiscale model for cornea transport into the tear balance is straightforward but a simpler model, presented below, captures the essential physics.

The mass balance for any solute dissolved in the tears, including drugs, is expressed as (see eq. 10 in [40]) 
$\frac{d\left(c_{\text {tear }} V_{\text {total }}\right)}{d t}=-c_{\text {tear }}\left(K_{\text {conjuctiva }} A_{\text {conjuctiva }}+K_{\text {cornea }} A_{\text {cornea }}\right)-c q_{\text {drainage }}$

where $\mathrm{K}_{\text {conjuctiva }}$ and $\mathrm{K}_{\text {cornea }}$ are the permeabilities through the conjunctiva and the cornea, respectively, $A_{\text {conjuctiva }}$ and $A_{\text {cornea }}$ are the areas of the conjunctiva and cornea in contact with the tear film, respectively, and $c_{\text {tear }}$ is the concentration of drug in the tear film [40]. Eq. 9 implicitly assumes that the transport across the cornea and the conjunctiva is at a pseudo-steady state. As discussed in Section 2, the transport of drugs through the cornea is highly complex and includes a slow accumulation over a few hours. This slow accumulation can be neglected while modeling drug delivery by eye drops due to the short residence time (approximately 5-10 min [40]), but even the time scale for transport across the stroma is of the order of tens of minutes suggesting that a pseudo-steady state approximation for transport across the cornea is not accurate. The detailed model for transport across the cornea described in Section 2 can be integrated into the drug transport model for eye drops [17] but, to keep the model simple, we present the results below only for the case where a pseudo-steady model is assumed. Similarly, these balance equations assume tears to be well-mixed. While other researchers have proposed models to account for spatial variations [52-55], including spatial variations does not change the basic physics and here we present only the simpler spatially uniform models. The mass balance equation (Eq. 9) can be solved easily by combining it with Eq. 8 to determine the concentration of drug in the tear fluid over time (see Eq. 11 in [40])

$\frac{c_{\text {tear }}(t)}{c_{0}}=\exp \left[-\int_{t_{0}}^{t} \frac{\left(K_{\text {conjuctiva }} A_{\text {conjuctiva }}+K_{\text {cornea }} A_{\text {cornea }}\right)+(1-\alpha) q_{\text {secretion }}}{V_{\text {total }}} d t\right]$ Eq. 10

where $\alpha$ is the fraction of tear film loss that is due to evaporation and absorption and $c_{0}$ is the concentration of drug in the tear film immediately after application of the eye drop. 
6.2 Model Validation - The model presented above can be used to estimate the corneal bioavailability of the drug, i.e., the fraction of the instilled drug that diffuses across the cornea. The bioavailability can be calculated as (see eq. 11 in [40])

$\beta=\frac{K_{\text {cornea }} A_{\text {cornea }}}{\left(V_{\text {tear }}+V_{\text {drop }}\right)} \int_{0}^{\infty} \frac{c_{\text {tear }}}{c_{0}} d t$

where $\beta$ is the bioavailability, $V_{\text {drop }}$ is the volume of the eye drop applied, and $V_{\text {tear }}$ is the total tear volume before addition of the eye drop [40]. The calculated bioavailability for the model drug timolol is around $1.16 \%$ [39] which is in reasonable agreement with reported data.

Tear drainage through the canaliculus contributes to drug loss from the tear film, and so it has been hypothesized that increasing the viscosity of the tear fluid to reduce the drainage rates could improve the bioavailability of the instilled drug. While this hypothesis is qualitatively valid, quantitative assessment using the models show only limited benefits of this approach. As discussed previously, increasing viscosity will reduce tear drainage rates only if the time scale for the compression of the canaliculi is longer than the blink time. Specifically, as the viscosity increases, the canaliculi requires more time to push the fluid out. Before a critical value of the viscosity, this time is less than the time for a typical blink phase (0.04s) resulting in only a small change in the drainage rate of the tear fluid caused by an increase in the viscous forces balancing the mechanical forces. However, if the viscosity is increased such that the compression time is longer than the blink phase, incomplete deformation of the canaliculi occurs resulting in a decrease in the drainage rate. This critical viscosity is estimated to be $4.4 \mathrm{mPa} \cdot \mathrm{s}$ [40]. The bioavailability of timolol was estimated when the viscosity was increased beyond this critical value, with the bioavailability increasing from $1.16 \%$ for viscosities below $4.4 \mathrm{mPa} \cdot \mathrm{s}$, to $1.20 \%$ for a viscosity of $100 \mathrm{mPa} \cdot \mathrm{s}$; a very small increase despite such a huge increase in viscosity [39]. 
The reason for this weak effect of viscosity can be understood by comparing the relevant time scales for drainage and transport across the epithelia. The absorption time scale, determined by $\mathrm{V} /\left(\mathrm{K}_{\text {conjuctiva }} \mathrm{A}_{\text {conjuctiva }}\right)$, is approximately $35 \mathrm{~s}$, while the time scale for drainage is close to $1000 \mathrm{~s}$. This shows that absorption (both through the cornea and through the conjunctiva) is the dominate method of removing drug from the tear film. Further, the permeability of the conjunctiva is three times higher than that of the cornea while having seventeen times the area of absorption of the cornea. Even assuming no drainage of the drug (for instance, by blocking the puncta somehow), the bioavailability can be calculated using (see eq. 20 in [39])

$\beta=\frac{K_{\text {cornea }} A_{\text {cornea }}}{K_{\text {cornea }} A_{\text {cornea }}+K_{\text {conjuctiva }} A_{\text {conjuctiva }}}$

Eq. 12

From this equation, the maximum bioavailability for the drug timolol is $1.34 \%$, only slightly above that for a highly viscous material, showing again that drainage of the drug through the canaliculi is not the dominate method of removal of the drug [39]. The relative benefits of increased viscosity would be significantly better for drugs with very low permeability for the ocular epithelia. Another situation where viscosity increase can impact the bioavailability is for hydrophobic drugs with low solubility that are formulated as emulsions such as the Restasis dry eye therapy which is an emulsion of cyclosporine dissolved in castor oil. For such systems the transport through the epithelia is proportional to the concentration of the dissolved drug while the drainage flux is proportional to the total concentration. Accordingly, a high fraction of the instilled drug is eliminated through the drainage pathway. Thus, slowing the drainage by increasing the viscosity could increase the bioavailability considerably [56].

6.3 Deficiencies of drug delivery by eye drops - The simplicity of developing formulations for delivery drugs to the eye using eye drops has caused eye drops to become the 
most commonly used method for treating eye conditions. However, the low bioavailability of the drugs for their target (estimated to be around 1\% for the models presented here with experiments showing $<5 \%$ ) results in the majority of the drug getting wasted and not reaching its intended target. In addition, the large fraction of the drug reaching the systemic circulation can cause many undesired side effects. The low corneal bioavailability from the eye drops results mainly from the large area of the conjunctiva compared to the cornea, which results in significantly larger drug transport through the conjunctiva into the blood, compared to the uptake into the cornea. It would thus seem that significantly improving the bioavailability must require the drug to have higher access to the cornea compared to the conjunctiva. A contact lens is therefore a natural choice for delivering drugs to the cornea.

\section{Drug delivery by contact lenses}

The anatomy relevant to ophthalmic drug delivery by contact lenses are illustrated in the schematic in Figure 9. In the absence of a contact lens, the eye is coated in a precorneal tear film. In addition to a high refractive index, this film serves to maintain an epithelium-tear interface, governing the hydration of the cornea and the transport across the cornea [57]. The addition of a contact lens divides the tear film into two different films, referred to as the pre-lens tear film (PLTF) and the post-lens tear film, or preocular tear film (POLTF). Delivery of therapeutic agents into the eye via contact lenses requires consideration of both of these films, as diffusion of the agent from the lens will occur into both the PLTF and the POLTF. The PLTF however breaks rapidly after eye opening limiting the flux of drug towards the PLTF. The rapid breakup also leads to partial dehydration of the film that can further reduce the transport towards the PLTF. The drug released from the lens towards the POLTF can either diffuse into the cornea or diffuse radially outwards into the tear lake. The radial diffusion is augmented by the periodic 
lens motion so the net flux in the radial direction can be characterized by the dispersion coefficient.

7.1 Modeling dispersive mixing in the POLTF - Creech et al. modeled the dispersive mixing in the post-lens tear film by solving the convection-diffusion equation using the convection obtained from the motion of the lens driven by the blink. The mass balance of any solute in the POLTF is given by the convective-diffusion equation (see eq. 3 in [52])

$\frac{\partial c}{\partial t}+v_{x} \frac{\partial c}{\partial x}+v_{y} \frac{\partial c}{\partial y}=D\left(\frac{\partial^{2} c}{\partial x^{2}}+\frac{\partial^{2} c}{\partial y^{2}}\right)$

Eq. 13

where $c(t, x, y)$ is the local solute concentration, $D$ is the molecular diffusion coefficient of the solute, and the longitudinal and transverse velocities are given by (see eqs. 1 and 2 in [52])

$v_{x}=\frac{6 V^{\prime} x}{h}\left(\zeta-\zeta^{2}\right) \cos \omega t+V \zeta \cos \omega t$

$v_{y}=V^{\prime}\left(2 \zeta^{3}-3 \zeta^{2}\right) \cos \omega t$

where $\zeta=y / h, V$ and $V^{\prime}$ represent the maximum longitudinal and transverse velocities, $v_{x}$ and $v_{y}$, respectively, $h$ is the thickness of the POLTF, and $\omega$ is the blink frequency. The longitudinal and transverse fluid flow is driven by the motion of the contact lens. The shear force exerted by the lids on the lens during the blink causes the lens to moves vertically [58]. Additionally, the pressure exerted by the lids on the lens during the blink, combined with the elastic recoil of the lens in the interblink drive the transverse motion of the lens [59]. Together with a no-flux boundary condition at the contact lens and cornea for the inert dye, Eq. 13 is solved under the approximations that transverse convection is small relative to longitudinal convection and that enough time has elapsed that the concentration profiles are locally linear. Normalizing and timeaveraging the concentration histories results in an averaged dispersion equation with the following expression for the effective dispersion coefficient, $D^{*}$, (see Eq. A9 in [52]) 
$D^{*}=D+\chi / 4 \omega \kappa^{2}$

Eq. 15

where $\kappa$ and $\chi$ are defined as (see eqs. A10 and A11 in [52])

$\kappa=\sqrt{\omega h^{2} / 2 D}$

Eq. 16a

$\chi=\left(4 a^{2}+6 a d+3 d^{2}\right) / 3+\left[\left(2 a^{2}+2 a d+d^{2}\right) \times(\cosh \kappa \sinh \kappa-\right.$

$\left.\cos \kappa \sin \kappa)+\left(2 a d+d^{2}\right) \times(\cosh \kappa \sin \kappa-\cos \kappa \sinh \kappa)\right] / \kappa\left(\cos ^{2} \kappa-\cosh ^{2} \kappa\right)$

Eq. $16 \mathrm{~b}$

where $a=-6 V^{\prime} x / h$ and $d=\left(6 V^{\prime} x / h\right)+V$.

7.2 Model Validation - The proposed dispersive-mixing theory was compared to experimental data collected by McNamara et al. [60]. In these experiments, 23 subjects wore four different types of contact lenses, and the mixing under the lens was observed by placing fluorescein isothiocyanate-dextran on the eye prior to lens insertion. The changes in fluorescence intensity were measured and quantified. The model presented by Creech very closely aligns with the experimental data for a theoretical POLTF thickness, $h$, of $15 \mu \mathrm{m}$.

7.3 Modeling drug delivery by contacts - The leading ophthalmic treatment, as discussed previously, is delivery of therapeutic agents via eye-drops which has only about 1-5\% corneal bioavailability. A contact lens is expected to provide an improved bioavailability due to its location in the proximity of the cornea. Thus contacts were proposed as potential drug delivery devices approximately five decades ago [61]. The relative improvements in bioavailability however were not quantified until $\mathrm{Li}$ and Chauhan developed a model for drug transport from a contact lens placed in an eye [62]. Their model involves a presoaked contact lens, where the lens is placed in a drug-containing solution and a concentration gradient drives drug uptake into the lens. The developed model predicts the release of this drug from the contact lens into the POLTF followed by subsequent uptake by the cornea, where treatment is desired. An idealized two-dimensional geometry between the PLTF, contact lens, POLTF, and cornea is 
adopted, in which the cornea and contact lens are undeformable and have negligible curvature, the POLTF is of constant thickness, and the effect of gravity is negligible in the POLTF.

The mass transfer of the drug released from the contact lens is coupled to its subsequent transport in the POLTF which involves a combination of radial dispersion and uptake by the cornea. The transport of drug from the contact lens towards the PLTF was considered in two extreme scenarios - Case I in which the flux into the PLTF is assumed to be zero, and Case II in which the concentration in the PLTF is assumed to be zero, which will lead to the maximum flux. The in vivo scenario is expected to lie in between these two cases. The governing equations for mass transfer in the tear film and lens are generated and can be solved by finite-difference or finite-element methods. However, it is possible to reduce the equations to a single integrodifferential equation that can be solved numerically. The dimensionless form of the integrodifferential equation is (see Eq. 14 in [62])

$\frac{\partial \tilde{C}_{f}}{\partial \tau}=P 1 \frac{\partial}{\partial \eta} \widetilde{D}^{*} \frac{\partial \tilde{C}_{f}}{\partial \eta}+P 2 \tilde{J}_{0}-P 3 \tilde{C}_{f}$ Eq. 17 where $P 1=\frac{D_{f} h_{g}{ }^{2}}{D_{g} L^{2}}, P 2=K \frac{h_{g}}{h_{i}}, P 3=\frac{k_{c} h_{g}{ }^{2}}{h_{i} D_{g}}, \tilde{J}_{0}$ is the dimensionless flux given from the contact lens to the tear film, $\mathrm{D}_{\mathrm{f}}$ is the drug diffusivity in the tear film, $\mathrm{h}_{\mathrm{g}}$ is the thickness of the twodimensional representation of the contact lens, $\mathrm{D}_{\mathrm{g}}$ is the drug diffusivity in the contact lens hydrogel matrix, $\mathrm{L}$ is the length of the two-dimensional representation of the contact lens, $\mathrm{K}$ is the ratio of the concentration of drug in the gel and in the POLTF at equilibrium, $\mathrm{h}_{\mathrm{i}}$ is the thickness of the POLTF, and $\mathrm{k}_{\mathrm{c}}$ is the mass transfer coefficient for drug transport into the cornea. The first term on the RHS represents the drug dispersion in the POLTF, while the second and the third terms represent the flux of drug from the lens into the film and that into the cornea, respectively. By solving the diffusion equation (see eqs. 15 and 16 in [62]) 


$$
\begin{array}{ll}
\tilde{J}_{0}=\left[2 \sum_{n=0}^{\infty} e^{-(2 n+1)^{2} \pi^{2} \tau / 4}+2 \sum_{n=0}^{\infty}\left(\int_{0}^{\tau} \tilde{C}_{f}\left(\tau^{*}\right) \frac{(2 n+1)^{2} \pi^{2}}{4} e^{\left[\frac{-(2 n+1)^{2} \pi^{2}}{4}\right]\left(\tau-\tau^{*}\right)} d \tau^{*}-C_{f}(\tau)\right)\right] & \text { Eq. 18a } \\
\tilde{J}_{0}=\left[\sum_{n=0}^{\infty} 4 e^{-(2 n+1)^{2} \pi^{2} \tau}-\tilde{C}_{f}(\tau)+2 \sum_{n=0}^{\infty}\left(\int_{0}^{2} \tilde{C}_{f}\left(\tau^{*}\right) n^{2} \pi^{2} e^{-n^{2} \pi^{2}\left(\tau-\tau^{*}\right)} d \tau^{*}-\tilde{C}_{f}(\tau)\right)\right] & \text { Eq. 18b }
\end{array}
$$

where $\tilde{\mathrm{C}}_{\mathrm{f}}$ is the dimensionless POLTF concentration, $\tau$ and $\tau^{*}$ are separate dimensionless times, $\eta$ is the dimensionless lateral position, $\tilde{\mathrm{D}}^{*}$ is the dimensionless effective dispersion coefficient (see previous section), and $\tilde{J}_{0}$ is the dimensionless flux of drug entering POLTF from lens. This model also neglects the detailed structure of the cornea for simplicity and quantified the transport as a pseudo-steady state with fixed permeability.

The drug transport is determined by three dimensionless parameters -- P1, P2, and P3. P1 is the ratio of diffusive time scales in the gel and the POLTF in the lateral direction, so increasing P1 will lead to an enhancement of dispersion. The thickness of the edge region will increase, and an increase in drug loss from the sides will be observed. P2 is the ratio of the amount of drug in the contact lens and the amount in the tear film at equilibrium, so increasing P2 is equivalent to increasing the partition coefficient of the solute. Since changes in P2 affect every axial location in the POLTF equally in case I, the fraction of drug delivered to the cornea remains relatively unchanged. In case II, however, there is a decrease in the fluxes to the cornea for an increase in P2 because the larger partition coefficient allows for more drug loss to the PLTF. P3 is the ratio of the time scale for diffusion in the lens and the time scale for drug uptake by the cornea. Increasing P3 is equivalent to an increase in the permeability of the cornea. Thus, increasing P3 increases the flux to the cornea, decreases the concentrations in the POLTF, and increases the fractional uptake by the cornea. The model developed by $\mathrm{Li}$ and Chauhan represents a first attempt at modelling the release of a therapeutic agent from a contact lens and its subsequent uptake into the cornea. It takes into account essential mechanisms of mass 
transfer but simplifies each of them. The model neglects more intricate details of contact lens application, such as the degree of swelling and interaction with the gel. This study showed that a contact lens, loaded with a drug via soaking, can significantly increase the bioavailability from 1$5 \%$ for eye drops to about $50-70 \%$, depending on the values of various parameters.

7.4 Model Validation - The model for ophthalmic drug delivery presented by Li and Chauhan was compared to a variety of clinical studies. A study performed by Hillman et al. suggests that drug delivery via contact lenses is $\sim 33$ times more efficient than delivery via eye drops [63]. Another study with the same type of lenses resulted in the same conclusion, an increase in efficiency by $~ 33$ times for lenses over eye drops [64]. The model presented by Li and Chauhan predicts that drug uptake by the contact lens to be $\sim 35$ times more effective than eye drops, which is in reasonable agreement with the clinical results.

7.5 Limitation of commercial contacts for drug delivery - Li notes that a soaked contact lens cannot deliver drugs for an extended period of time. Implementing an approach for increasing the delivery duration of a therapeutic agent allows for more control over the delivery of said agent. Delivering too much of a drug in a short amount of time can result in toxicity, and a treatment cycle could require more than a single lens in a day. Thus, extending the release duration of a therapeutic agent from a contact lens could help reduce toxicity while delivering an adequate amount of said agent in a single treatment cycle.

7.6 Approaches for improving drug release - Peng et al. create transport barriers in the lens by loading the lens with Vitamin E, forcing drug molecules to diffuse along a tortuous path [65] (Figure 10). The barriers are created in pre-fabricated contact lenses by soaking the lenses in a solution of vitamin $E$ in ethanol followed by ethanol extraction in water. Since vitamin $E$ is insoluble in water, it is trapped in the lens, leading to precipitation likely along the phase 
boundaries between the silicone and the hydrophilic phases of the lenses. The nanosized barrierloaded lenses remain transparent but increase the tortuosity for transport of hydrophilic drugs. The effect of Vitamin E on the release duration of timolol in a lens is shown in Figure 11. A pure lens, with no Vitamin E loading, releases $90 \%$ of the drug in about an hour. This duration can increase to as much as 225 hours with $75 \%$ Vitamin E loading, though wearing a contact lens for such a duration would prove infeasible. A more practical loading is the $16 \%$ Vitamin $\mathrm{E}$ loading where the $90 \%$ drug release duration is $\sim 8$ hours. The drug transport in a vitamin $\mathrm{E}$ loaded contact lens can be modeled as 1-D diffusion with an attenuated effective diffusivity that depends on the vitamin E loading fraction $\phi$. The attenuated effective diffusivity is given by (derived from Eq. 3 in [65])

$D_{\text {attenuated }}=D\left\{\left(1+\frac{\phi}{3}\right)^{2}\left[1+\alpha\left(\phi-\phi^{*}\right)\right]^{2}\right\}^{-1}$

where $D$ is the effective diffusivity, $\phi$ is the volume ratio of vitamin $\mathrm{E}$ in the dry gel, $\phi^{*}$ is the volume fraction of vitamin $\mathrm{E}$ that does not contribute to drug transport, and $\alpha$ is a parameter that depends on the microstructure of the vitamin $\mathrm{E}$ aggregates in the gel. The barrier mechanism was proven by a number of indirect approaches including showing that the relative decrease in drug diffusivity is similar for many hydrophilic drugs, and depends on the square of the vitamin E loading, which is consistent with the barrier model. The safety and efficacy of the vitamin E loaded lenses was proven by in-vivo studies in which glucomatous beagle dogs were treated for glaucoma using contact lenses loaded with timolol [66]. Incorporation of vitamin E resulted in an increased release duration from a few hours to several days, with no sign of discomfort or ocular toxicity in the dogs. Furthermore, the lens based therapy achieved comparable intraocular pressure reduction as eye drops with significantly lower drug loading. Several properties of contact lenses have been tested to ensure the loading of Vitamin E does not affect lens 
detrimentally. Ion permeability is critical for lens motion on the eye [67], so the ion permeability of various lenses was calculated for a range of Vitamin E loading. As expected, the ion permeability decreases with increasing Vitamin E loading; however, the Vitamin E loaded lenses maintain adequate ion permeability for sufficient on-eye movement. Oxygen permeability is similarly important, as depriving the cornea of oxygen can cause adverse responses. The suggested minimum value of oxygen transmissibility to avoid hypoxia is proposed to be $34.3 \mathrm{x}$ $10^{-9} \mathrm{~cm} \mathrm{x} \mathrm{ml} \mathrm{O}_{2} \mathrm{x} \mathrm{s}^{-1} \mathrm{x} \mathrm{ml}^{-1} \mathrm{x} \mathrm{mmHg}$ [68]; each lens investigated exceeded this value, indicating no detrimental effect on oxygen permeability by the addition of Vitamin E diffusion barriers. In fact, Vitamin E loading has a greater influence on ion permeability than oxygen permeability, suggesting that most Vitamin E aggregates don not block transport through the silicone phase. Finally, the transmittance of a Vitamin E loaded contact lens was investigated, as exposing the corneal tissue to UV light has damaging effects [69]. While some commercial contact lenses claim to have protection from UV radiation, such as ACUVUE ${ }^{\circledR}$, other lenses have no significant UV blocking capabilities, such as NIGHT\&DAY ${ }^{\mathrm{TM}}$ and $\mathrm{O}_{2} \mathrm{OPTIX}^{\mathrm{TM}}$. The effect of Vitamin E loading on the transmittance spectrum showed that Vitamin E loading completely blocks UVB radiation in NIGHT\&DAY ${ }^{\mathrm{TM}}$ lenses, and increases the UV blocking capabilities in ACUVUE ${ }^{\circledR}$, $\mathrm{s}$ existing UV blocking lenses.

While vitamin E has proven quite effective as a diffusion barrier, providing more control over the release of drugs from contact lenses, it is not the only method proposed. Gulsen et al. suggest using nanoparticle laden gels, in which the nanoparticles are loaded with a therapeutic agent. The nanoparticles can be released at a controlled rate [70]. Additionally, Venkatesh et al. propose the use of biomimetic carriers for drug delivery. In-vitro, these gels can release a therapeutic agent in a controlled fashion for 5 days, with the potential to increase that dosage if a 
protein is incorporated [71]. These methods are promising, but focus on hydrophilic hydrogel based contact lenses, which have limited oxygen permeability not conducive for extended wear.

\section{Conclusions}

The human eye is a fascinating system for researchers interested in interfacial phenomena and transport, and there is potential to further improve the understanding of the physiology by developing systems based models. As an example, we have focused on understanding the dynamics of tears by first focusing on all relevant transport pathways individually, and then focusing on integration into a combined model to predict the tear dynamics. The individual models for transport across the cornea and the conjunctiva and the model for tear drainage were validated by comparison with experiments. All models were based on physiology and only used physical parameters that are available in literature or can be measured separately. Predictions from the models were in reasonable agreement with a majority of the available data. Individual models were then combined into a tear dynamics model that can predict the steady state volume, composition, and potential, as well as the dynamics following instillation of eye drops. Again, the model predictions for the steady state, as well as the dynamics, were in good agreement with reported data in literature. This model can be very useful in testing hypotheses, e.g., whether conjunctiva secretes or absorbs fluid, whether electro-osmotic water transport is important in eyes, whether certain drugs that modify ion-transport could increase tear secretion, and many others. It can also predict the loss of instilled drugs through various pathways and compute the portion that reaches the target tissue. The predictions for corneal bioavailability were in good agreement with reported data in literature. Further, the model also suggests that the bioavailability could be significantly increased by using a device that is located near the cornea, 
motivating the use of drug eluting contacts. Mathematical modeling of drug delivery by contact lenses suggested that approximately $50 \%$ of the loaded drug could reach the cornea, compared to $<5 \%$ for eye drops. This prediction was supported by clinical studies and recent in vivo animal studies. While contacts are optimal devices for corneal drug delivery, commercial lenses were not optimized for drug transport. Simple modifications such as the incorporation of nanoparticles or nanobarriers can improve the drug release profiles without impacting any other critical property. The in vivo animal studies with drug eluting contacts have shown very promising results.

While the models discussed here are useful, there is a potential for further improvements. For example, spatial gradients were neglected here, though they have been incorporated into models by other researchers. Further, the detailed mechanisms of transport across the cornea were not considered in the tear dynamics or the drug delivery models. The model for drug delivery by contacts can be further improved by including the entire eye instead of only considering the tear film. There are several important questions that could potentially be addressed by combining detailed models with measured data, e.g., can contacts or eye drops deliver drug effectively to the back of the eye. We continue to focus on some of these problems and hopefully this review will encourage other researchers into this area.

Acknowledgements - The work in this paper was partly funded by the National Science Foundation. 


\section{Reference List}

[1] Urtti,A, L Salminen, Surv. Ophthalmol., 1993; 37: 6.

[2] Achouri,D, K Alhanout, P Piccerelle, V Andrieu, Drug Dev. Ind. Pharm., 2012;00.

[3] Hsu,K-, S Gause, A Chauhan, Journal of Drug Delivery Science and Technology, 2014; $24: 2$.

[4] Bengani,LC, K Hsu, S Gause, A Chauhan, Expert opinion on drug delivery, 2013;0.

[5] Liu,S, L Jones, F X Gu, Macromolecular Bioscience, 2012; 12: 5.

[6] Rawas-Qalaji,M, C Williams, Curr Eye Res, 2012; 37: 5.

[7] Gan,L, J Wang, M Jiang, H Bartlett, D Ouyang, F Eperjesi, J Liu, Y Gan, Drug Discov. Today, 2013; 18: 5-6.

[8] Mishima,S, A Gasset, S D Klyce Jr., J L Baum, Invest. Ophthalmol. Vis. Sci., 1966; 5: 3.

[9] Watsky,MA, M M Jablonski, H F Edelhauser, Curr. Eye Res., 1988; 7: 5.

[10] DM Maurice, S Mishima. M.L. Sears (Ed.), Pharmacology of the Eye, Springer Berlin Heidelberg; 1984. Ocular Pharmacokinetics.

[11] Ahmed,PTF, Invest. Ophthalmol. Vis. Sci., 1985; 26: 4.

[12] Gaudana,R, H K Ananthula, A Parenky, A K Mitra, AAPS J., 2010; 12: 3.

[13] Reinstein,DZ, T J Archer, M Gobbe, R H Silverman, D Jackson Coleman, J. Refract. Surg., 2009; 25: 9.

[14] Srinivas,SP, Optom. Vis. Sci., 2010; 87: 4.

[15] McLaren,JW, N Ziai, R F Brubaker, Exp. Eye Res., 1993; 56: 3.

[16] Amrite,AC, H F Edelhauser, U B Kompella, Invest. Ophthalmol. Vis. Sci., 2008; 49: 1.

[17] Gupta,C, A Chauhan, R Mutharasan, S P Srinivas, Pharmaceut. Res., 2010; 27: 4.

[18] Gupta,C, A Chauhan, S P Srinivas, Pharmaceut. Res., 2012; 29: 12.

[19] Liu,X, B Testa, A Fahr, Pharmaceut. Res., 2011; 28: 5.

[20] Oba,Y, S R Poulson, Geochemical Journal, 2012; 46: 6. 
[21] Mannermaa,E, K Vellonen, A Urtti, Adv. Drug Deliv. Rev., 2006; 58: 11.

[22] Sugano,K, M Kansy, P Artursson, A Avdeef, S Bendels, L Di, G F Ecker, B Faller, H Fischer, G Gerebtzoff, H Lennernaes, F Senner, Nat. Rev. Drug. Discov., 2010; 9: .

[23] Cholkar,K, S P Patel, A D Vadlapudi, A K Mitra, J. Ocul. Pharmacol. Ther., 2013; 29: 2.

[24] Wallace,DG, J Rosenblatt, Adv. Drug Deliv. Rev., 2003; 55: 12.

[25] Hsu,K, B E Carbia, C Plummer, A Chauhan, European Journal of Pharmaceutics and Biopharmaceutics, 2015; 94: .

[26] Nichols,BA, Microsc. Res. Tech., 1996; 33: 4.

[27] Gipson,IK, Exp. Eye Res., 2004; 78: 3.

[28] Govindarajan,B, I K Gipson, Exp. Eye Res., 2010; 90: 6.

[29] Bron,AJ, P Argüeso, M Irkec, F V Bright, Prog. Retin. Eye Res., 2015; 44: .

[30] Kompella,UB, K Kim, V H L Lee, Curr Eye Res, 1993; 12: 12.

[31] Shi,X, O A Candia, Curr Eye Res, 1995; 14: 10.

[32] Levin,MH, J K Kim, J Hu, A S Verkman, Invest. Ophthalmol. Vis. Sci., 2006; 47: 1.

[33] Zhu,H, A Chauhan, Curr. Eye Res., 2007; 32: 3.

[34] Maurice,DM, Int Ophthalmol Clin, 1973; 13: 1.

[35] Rosengren,B, Ophthalmologica, 1972; 164: .

[36] H. Gray, Anatomy of the Human Body, Philadelphia: Lea \& Febiger, 1918.

[37] Doane,MG, Ophthalmology, 1981; 88: 8.

[38] Zhu,H, A Chauhan, Curr. Eye Res., 2005; 30: 8.

[39] Zhu,H, A Chauhan, Optom. Vis. Sci., 2008; 85: 8.

[40] Zhu,H, A Chauhan, Curr. Eye Res., 2005; 30: 10.

[41] Zhu,H, S Bhatia, A Chauhan, Curr. Eye Res., 2007; 32: 10.

[42] Wilson,G, R Merrill, Am. J. Optom. Physiol. Opt., 1976; 53: 2. 
[43] Cerretani,CF, C J Radke, Curr. Eye Res., 2014; 39: 6.

[44] Leung,BK, J A Bonanno, C J Radke, Prog. Retin. Eye Res., 2011; 30: 6.

[45] Ishibashi,T, N Yokoi, A J Bron, J M Tiffany, A Komuro, S Kinoshita, Curr. Eye Res., 2003; 27: 2 .

[46] Yokoi,N, A Komuro, Exp. Eye Res., 2004; 78: 3.

[47] Mishima,S, D M Maurice, Exp. Eye Res., 1961; 1: 1.

[48] Snibson,GR, J L Greaves, N D W Soper, J I Prydal, C G Wilson, A J Bron, Eye, 1990; 4: .

[49] Wilson,CG, Y P Zhu, M Frier, L S Rao, P Gilchrist, A C Perkins, British Journal of Ophthalmology, 1998; 82: 10.

[50] Scuderi,AC, A De Lazzari, F Miano, P Zola, Cornea, 2002; 21: 1.

[51] Meseguer,G, P Buri, B Plazonnet, A Rozier, R Gurny, J. Ocul. Pharmacol. Ther., 2009; 12: 4.

[52] Creech,JL, A Chauhan, C J Radke, Ind Eng Chem Res, 2001; 40: 14.

[53] Braun,RJ, Annu. Rev. Fluid Mech., 2012; 44: 1.

[54] Braun,RJ, A D Fitt, Mathematical Medicine and Biology, 2003; 20: 1.

[55] Gaffney,EA, J M Tiffany, N Yokoi, A J Bron, Prog. Retin. Eye Res., 2010; 29: 1.

[56] Gupta,C, A Chauhan, J. Controlled Release, 2011; 150: 1.

[57] Holly,FJ, Exp. Eye Res., 1973; 15: 5.

[58] Chauhan,A, C J Radke, Curr. Eye Res., 2001; 22: 2.

[59] Chauhan,A, C J Radke, J. Colloid Interface Sci., 2002; 245: 1.

[60] McNamara,NA, K A Polse, R J Brand, A D Graham, J S Chan, C D McKenney, Am. J. Ophthalmol., 1999; 127: 6.

[61] WICHTERLE,O, D LÍM, Nature, 1960; 185: 4706.

[62] Li,C, A Chauhan, Ind Eng Chem Res, 2006; 45: 10.

[63] Hillman,JS, J B Marsters, A Broad, Trans. Ophthalmol. Soc. U. K., 1975; 95: 1. 
[64] Hillman,JS, Br J Ophthalmol., 1974; 58: 7.

[65] Peng,C, J Kim, A Chauhan, Biomaterials, 2010; 31: 14.

[66] Peng,C, A Ben-Shlomo, E O Mackay, C E Plummer, A Chauhan, Curr. Eye Res., 2012; 37:

3.

[67] Austin,D, R V Kumar, Ionics, 2005; 11: 3-4.

[68] Holden,BA, G W Mertz, Invest. Ophthalmol. Vis. Sci., 1984; 25: 10.

[69] Pitts,DG, A P Cullen, P D Hacker, Invest. Ophthalmol. Vis. Sci., 1977; 16: 10.

[70] Gulsen,D, C Li, A Chauhan, Curr. Eye Res., 2005; 30: 12.

[71] Venkatesh,S, S P Sizemore, M E Byrne, Biomaterials, 2007; 28: 4.

\section{Figure captions}

Figure 1: Illistration of the tear film in a human eye.

Figure 2. Schematic illustration of the simplified cornea structure stratified into three layers: the epithelium, the stroma and the endothelium.

Figure 3. Trans-corneal penetration of rhodamine across rabbit cornea. Rhodamine was infused from the tear at a constant concentration. $\mathrm{Y}$-axis represents fluorescence in arbitrary units (AU). Circle dots represents the in vitro experiment data measured by using a custom-built scanning microfluorometer at time 6, 30, 60 and 140 minutes. The solid lines are the best fit from the model predictions. Reproduced from [17].

Figure 4. Transport across the cellular layers: The main mechanism for transport of lipophilic solutes is through the lipid bilayers of the plasma membrane (dark arrow). Another important but slower mechanism leads to continued accumulation of hydrophobic solute in the intracellular hydrophobic domains (e.g., membrane associated with endoplasmic reticulum). This mechanism can be resolved into following steps in sequence: (1) transport by partition into the bilayer of the plasma membrane from tears, (2) partitioning of the drug into cytoplasm from the bilayer, and (3) partitioning into intracellular hydrophobic domains. Step 2' and 3' represent steps analogous to the required 2 and 3 , which permit an alternative route for transcellular movement of the lipophilic solutes. Hydrophilic solutes mainly pass through paracellular pathways (dotted arrow) due to the significant resistance encountered in the lipid bilayers. However, hydrophilic solutes can also pass through transcellular pathways and get accumulated in the cell if the residence time in the tear is long enough. Reproduced from [17].

Figure 5. (a) Transient concentration profile of fluorescein across the rabbit cornea, when the endothelium side was exposed to a fixed concentration of the dye; $\mathrm{Y}$-axis represents fluorescence in arbitrary unit (AU). The fluorescence scans were obtained with a custom-built scanning microfluorometer. (b) Comparison of the model predictions and the experimental profiles of the fluorescein concentration in the stroma for short durations $(t<$ $3 \mathrm{hr}$ ) using the best-fit values of the parameters. Here depth $384 \mu \mathrm{m}$ represents the epithelium-stroma interface while depth $50 \mu \mathrm{m}$ represents the stroma-endothelium interface. The time at which the profiles are compared is indicated on each curve. Reproduced from [18].

Figure 6. Intraocular pressure (IOP) measurement of 10 Beagle dogs with treatment of timolol and dorzolamide loaded contact lenses modified with $20 \%$ vitamin E diffusion barriers. For each dog, only one randomly chosen eye 
( 5 in left eyes and 5 in right eyes) was receiving treatment and the other eye was untreated as a control. The dash line at 48 hours indicates the time the contact lenses were replaced with a fresh one. The other dash line at 96 hours indicates the time the contact lenses were removed. No lens was on the eye during 96 to 288 hours. No data was collected during 120 to 168 hours. Data are presented as mean \pm SEM. The IOP was significantly reduced in the treated eyes due to the effect of the glaucoma drugs. The therapeutic effects sustained for more than a week even after the drug-eluting contact lenses were removed at 96 hours. Reproduced from [25].

Figure 7: Simplified illustration of the structure of and transport across the conjunctiva. Reproduced from [41].

Figure 8: Illustration of the pumping action between the blink (a) and interblink (b) phases for the canaliculi. Reproduced from [38]. (c) The structure of the lacrimal system is shown with the lacrimal ducts here refer to the canaliculi. Reproduced from [36].

Figure 9: Schematic of a contact lens placed upon the eye

Figure 10: Illustration of the effect of vitamin E on the diffusion of drug molecules

Figure 11: Increase in drug release time as a function of $\phi$, the volume fraction of Vitamin $E$ loaded into the lens, for three different drugs in ACUVUE $^{\circledR}$ OASYS ${ }^{\mathrm{TM}}$ contact lenses. Source reproduced from [65] 


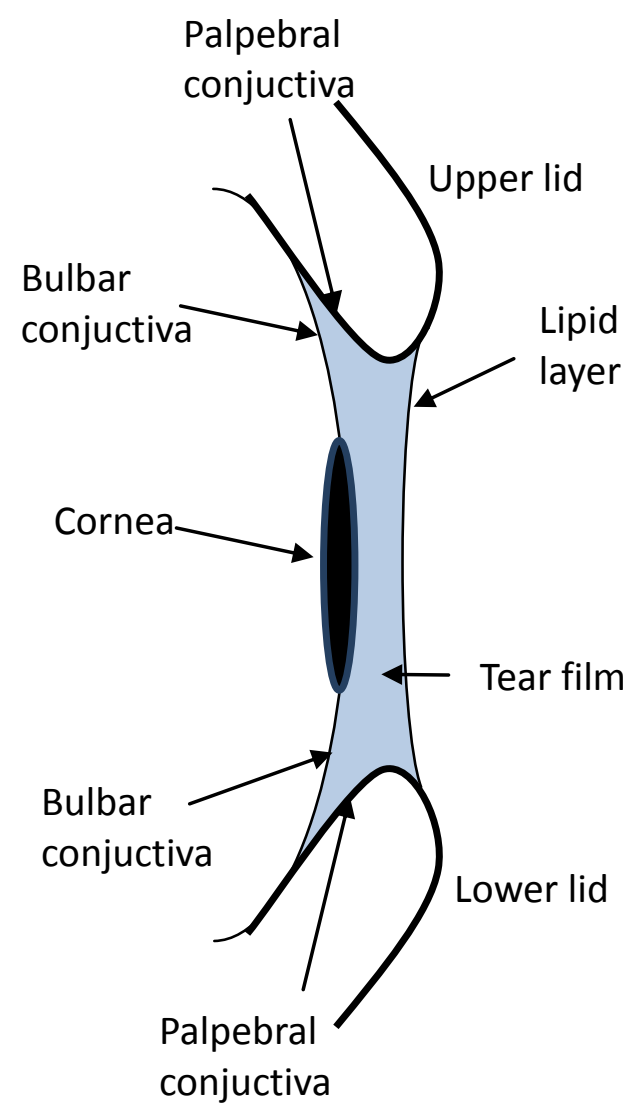

Palpebral 


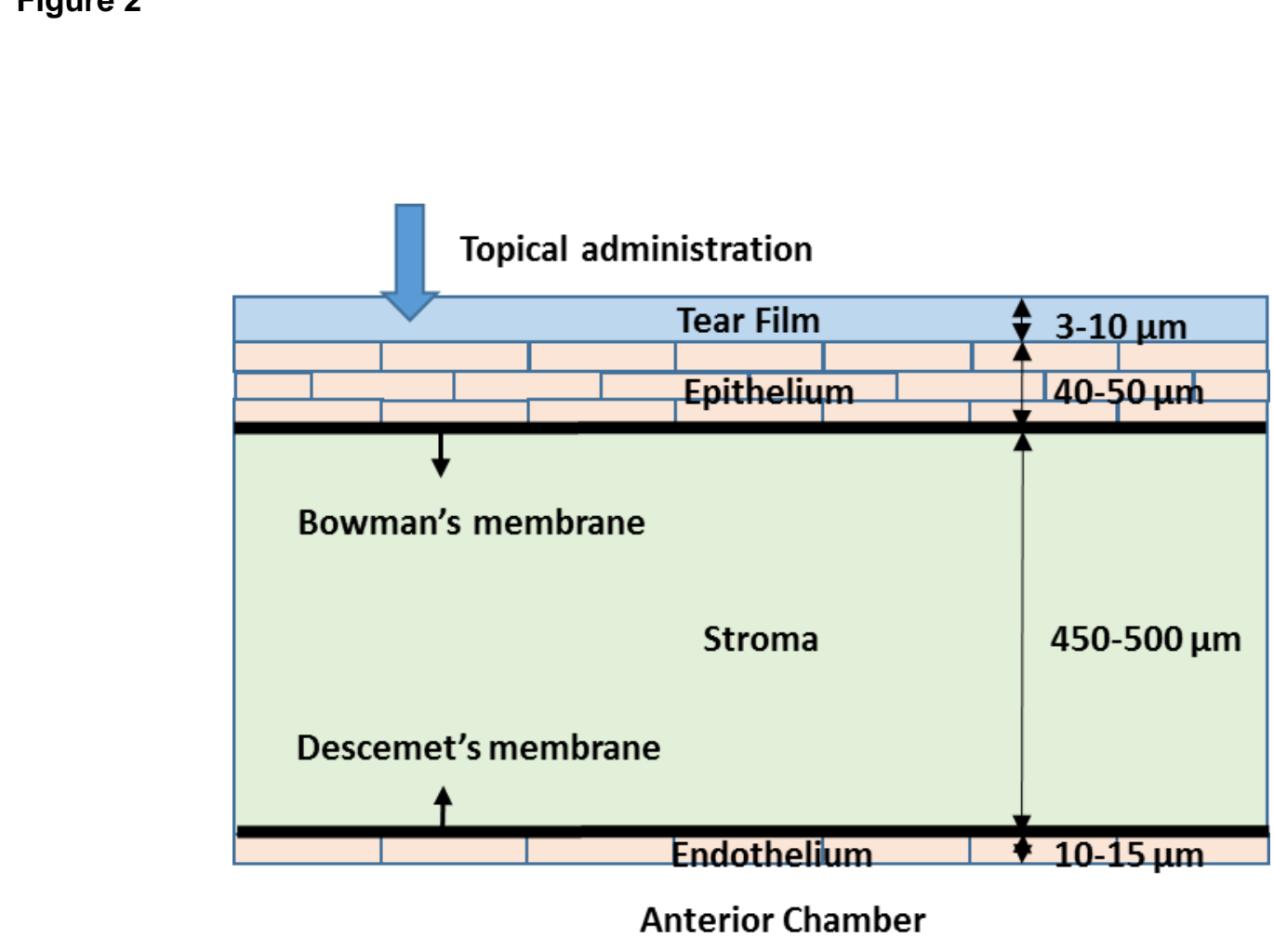

Figure 2

\section{Anterior Chamber}

2

\section{Anterior Chamber}

Figure 
(A)
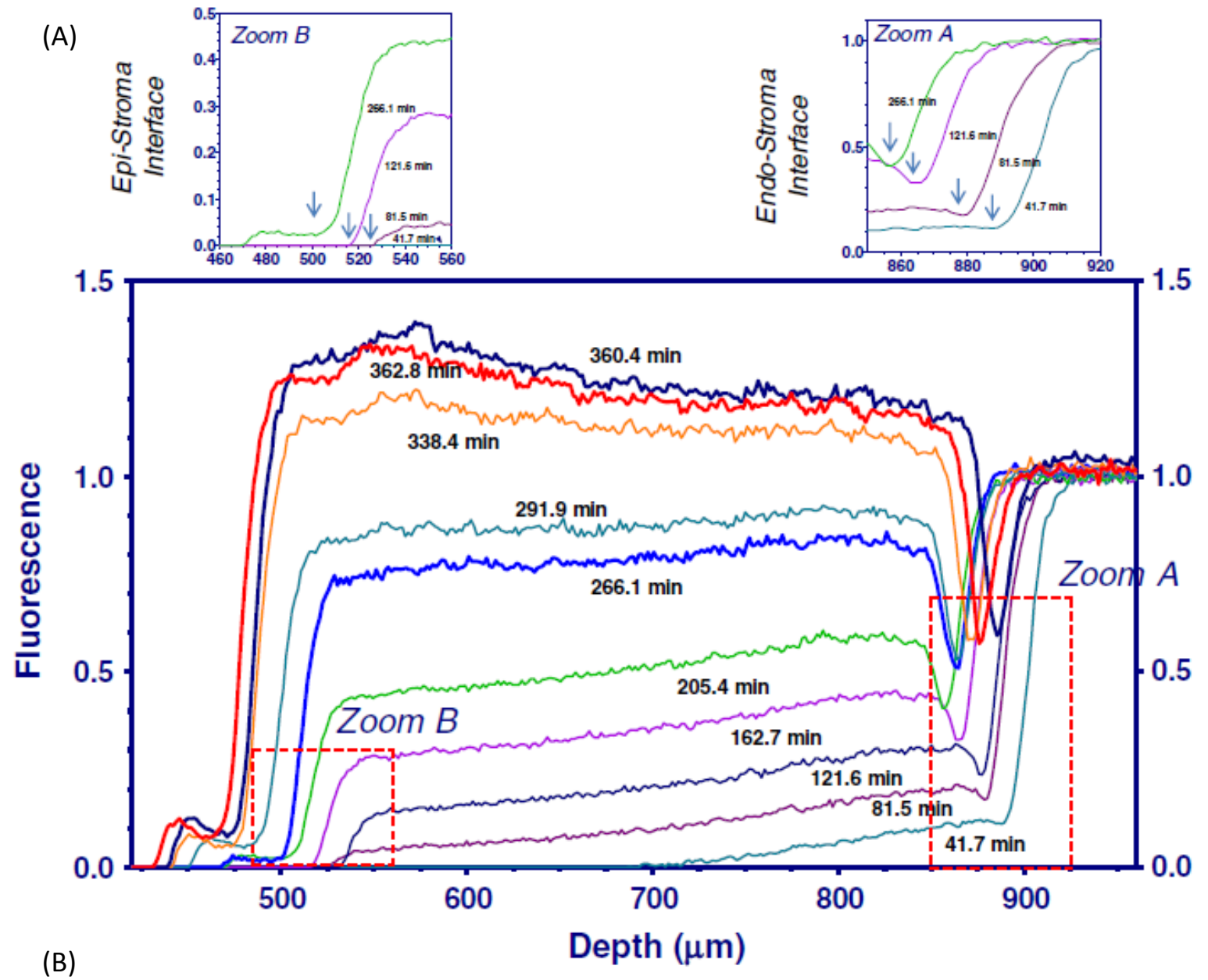

(B)

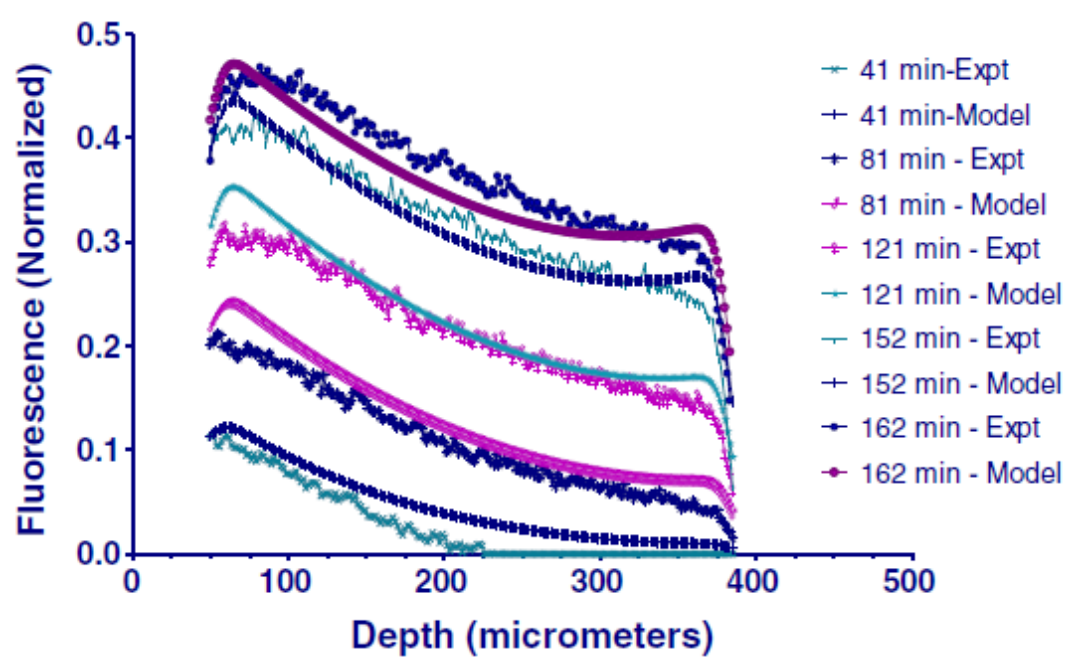


Figure 6

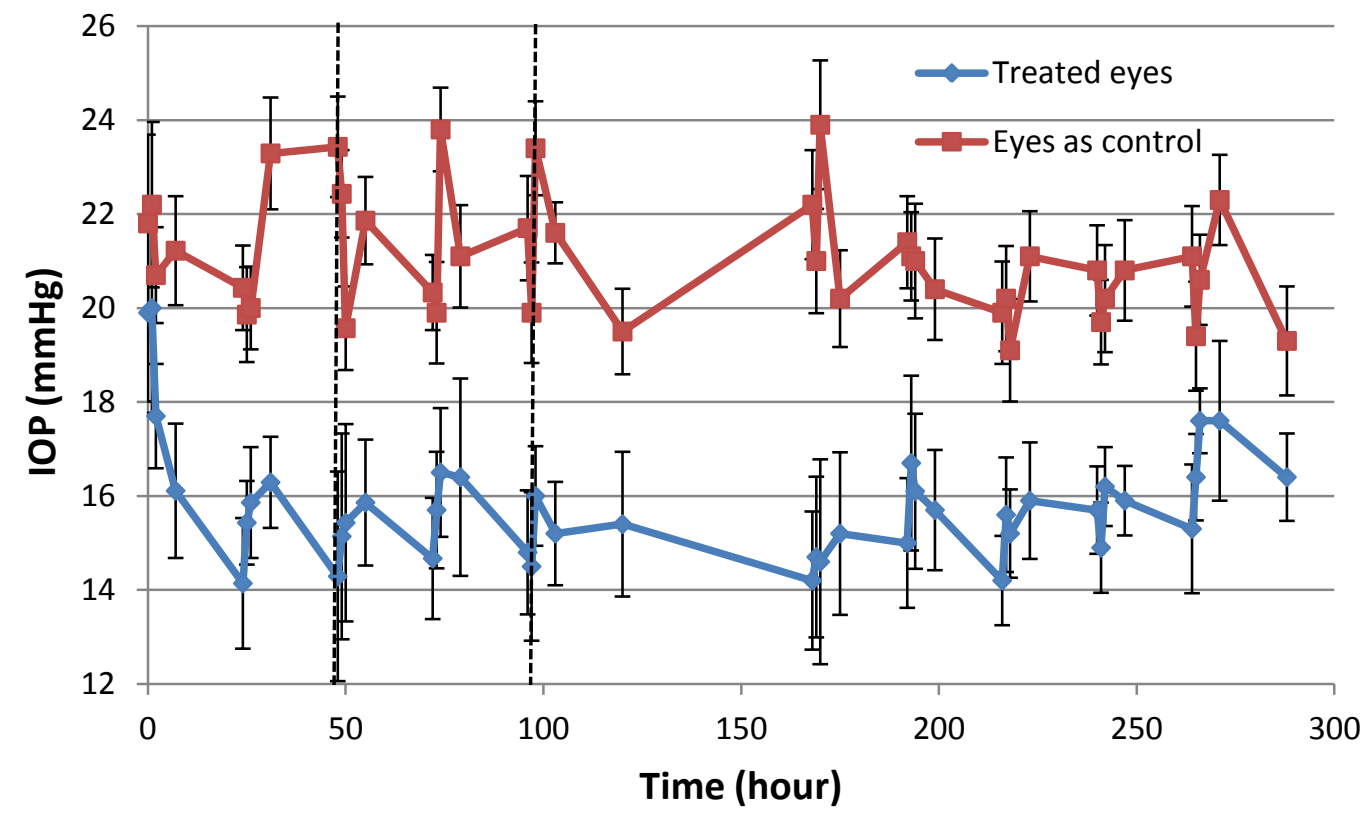


Figure 7
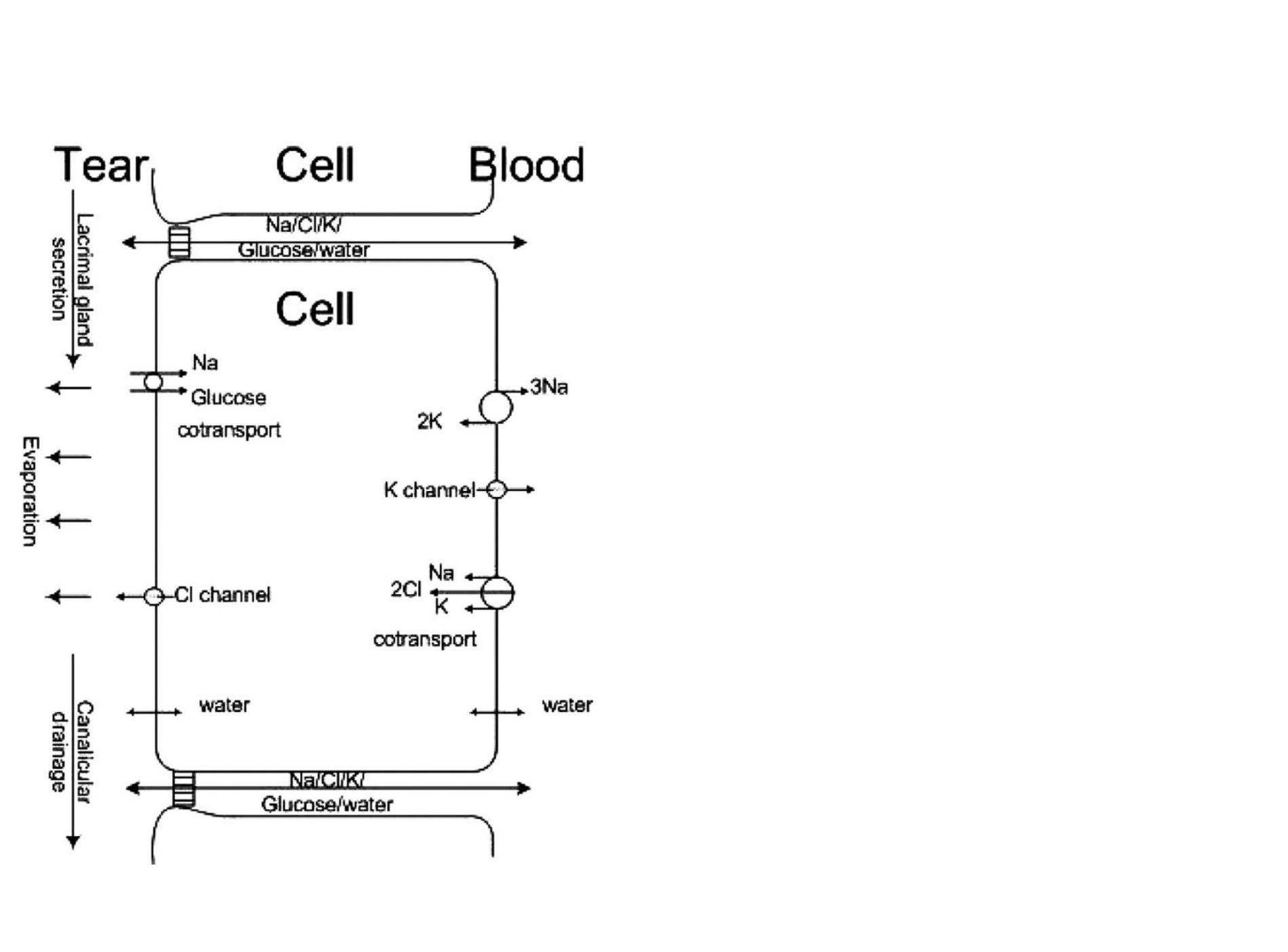
(a)

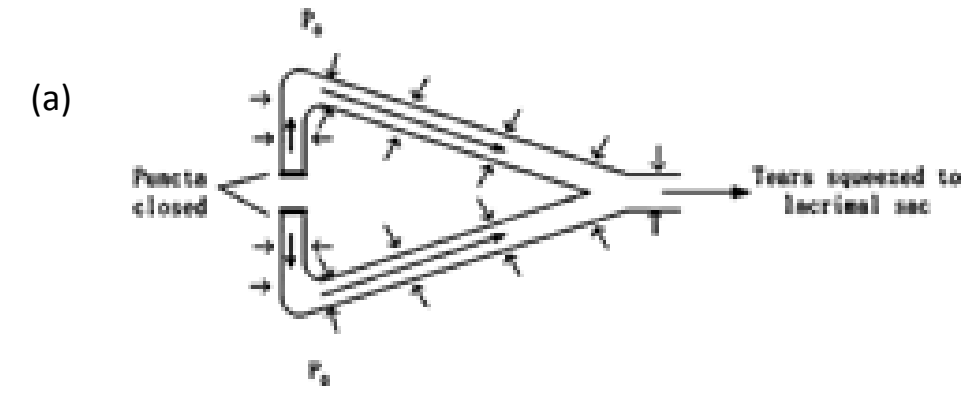

$P_{8}$

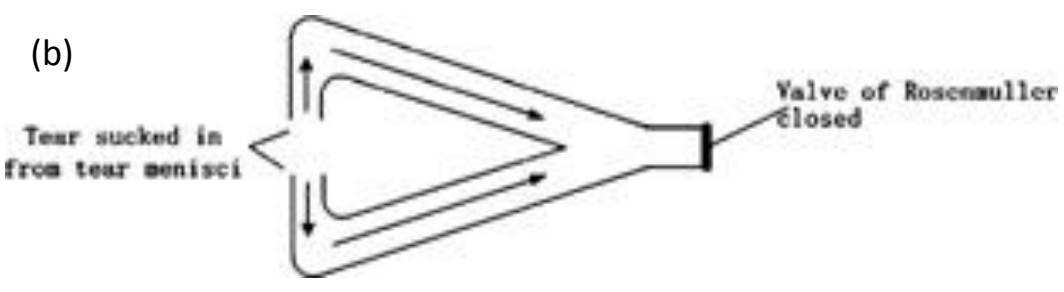

(c)

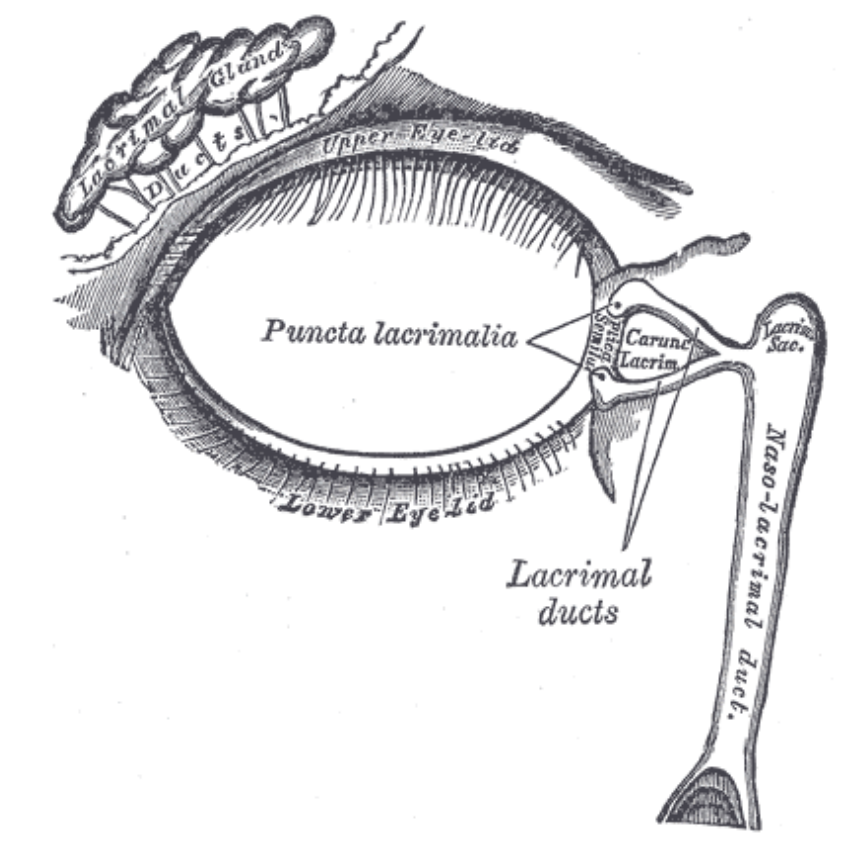


Figure 9
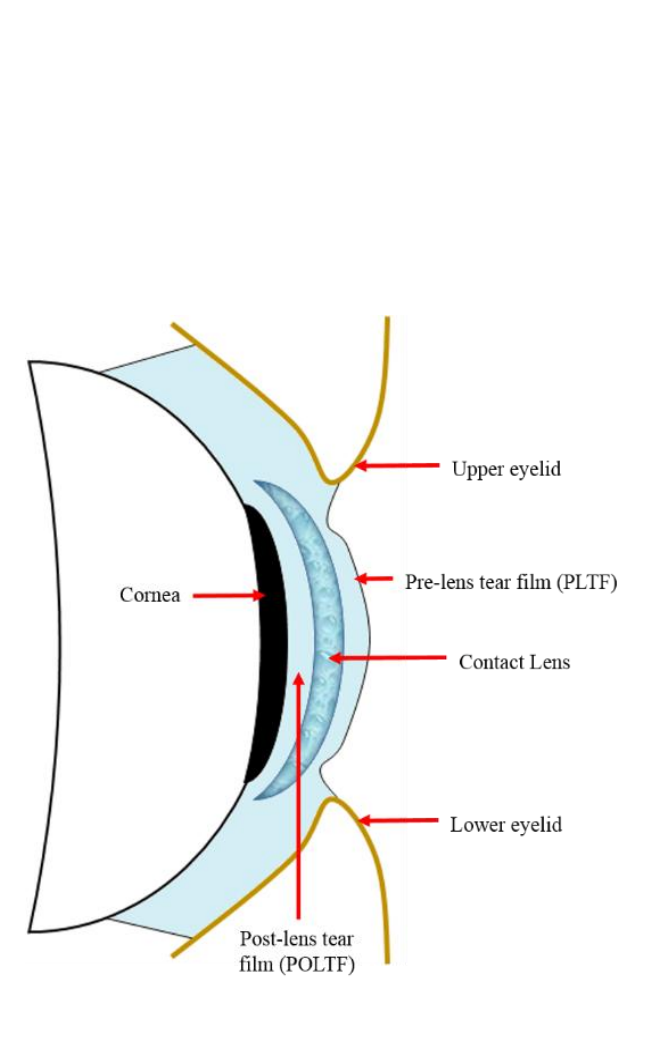


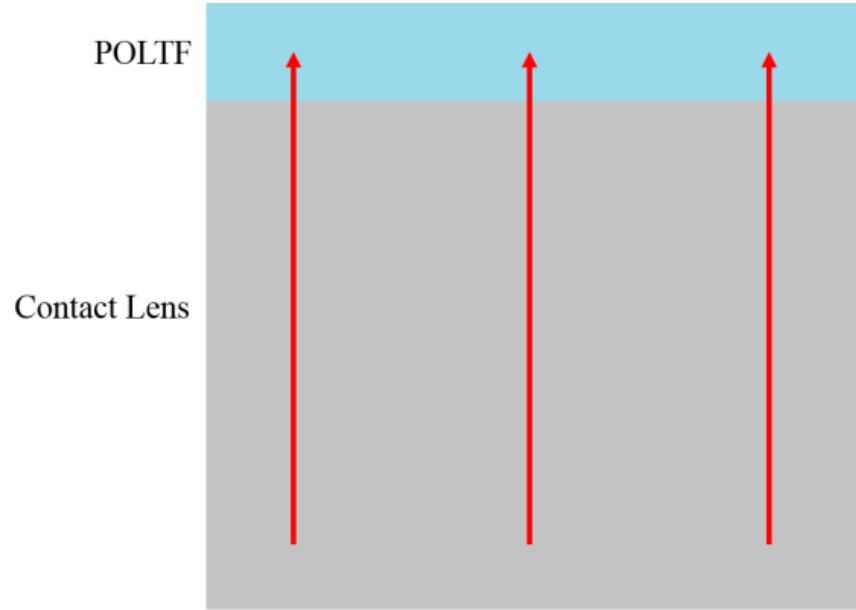

Without vitamin E barriers

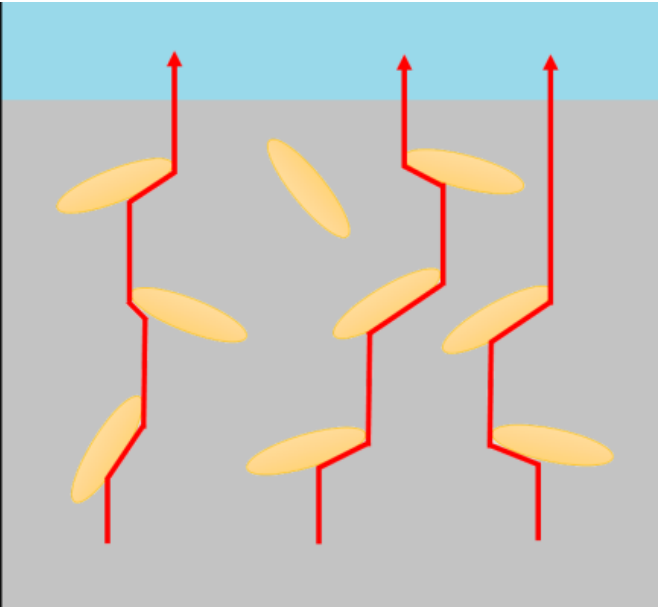

With vitamin E barriers 


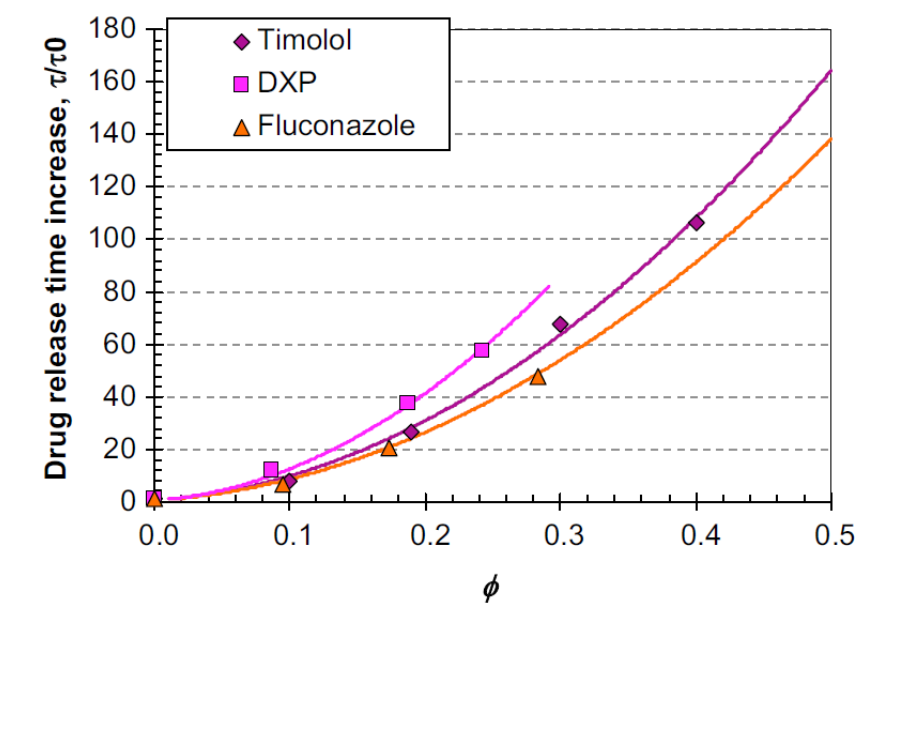

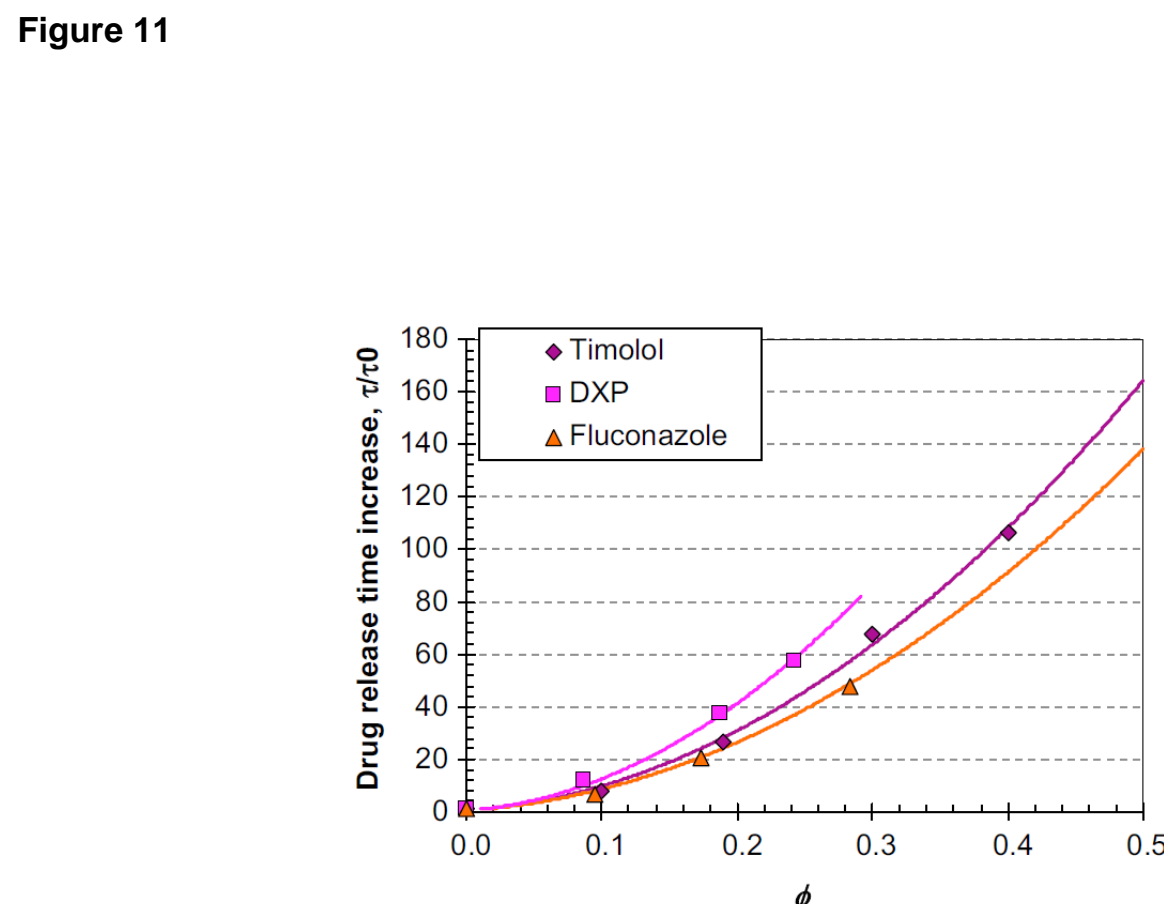

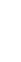

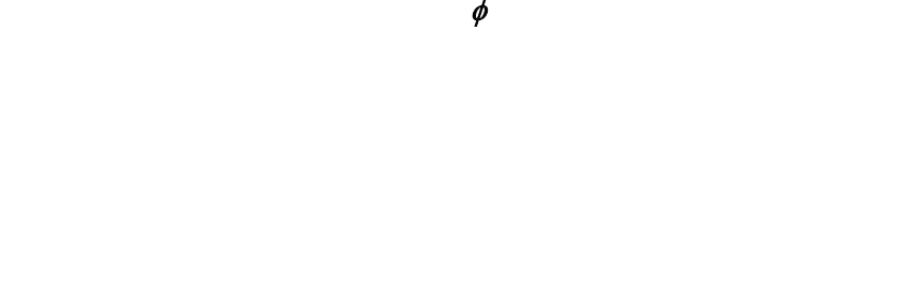

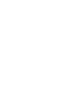
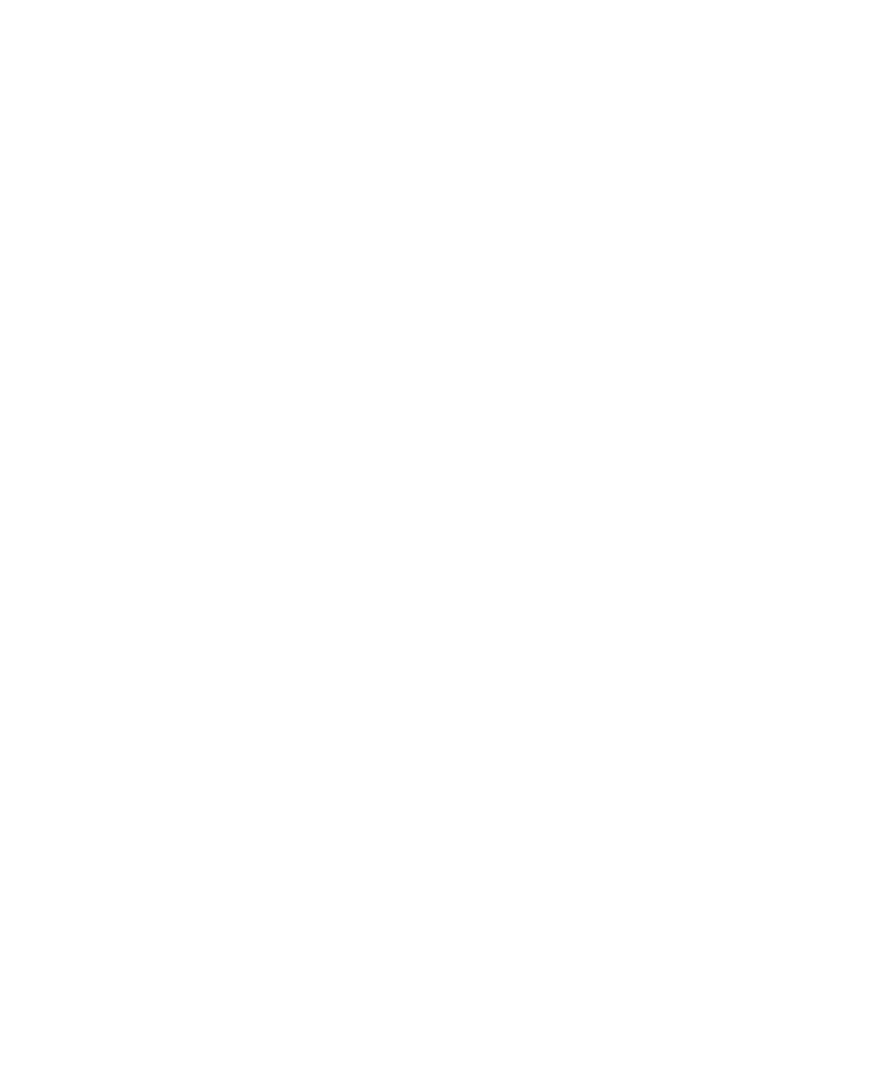